\title{
A STUDY OF THE BEHAVIOR OF THE WHITE RAT BY THE MULTIPLE CHOICE METHOD
}

\author{
HAROLD E BURTT \\ From the Psychological Laboratory of Harvard University \\ INTRODUCTION
}

The multiple choice method of studying ideational and allied forms of behavior has already been described in connection with reports of results. ' It consists essentially in presenting to a subject of any type, age, or condition a problem "which may be solved by the perception of a certain constant relation or group of relations within the reaction-mechanisms." For instance the subject may be required to operate the right-hand mechanism or the second from the left in a group of mechanisms varying in number from two to nine.

In this way the same problem can be presented to widely varied organisms, it being necessary merely to adapt the form of reaction mechanism to the species of animal. For human subjects a simple keyboard suffices,- - the sounding of a buzzer as indication of a correct choice proving ample incentive to effort. With crows, pigs, ring-doves, rats and monkeys a series of similar boxes, each with an entrance and an exit door, has been employed, with confinement as punishment for incorrect choices and food as reward for correct choices. The value of this method from the comparative standpoint is obvious.

\section{SUBJECTS}

Three outbred and two inbred albino rats were used in the experiments. All were born in the Harvard Psychological Lab633.

${ }^{1}$ Yerkes, Robert M. The study of human behavior. Science, 1914, 39, 625-

Coburn, Charles A. and Yerkes, Robert M. A study of the behavior of the crow, Corvus Americanus Aud., by the multıple-choice method. Journal of Animal Behavior, $1915,5,75-114$.

Terkes, Robert M. and Coburn, Charles A. A study of the behavior of the pig, Sus scrofa, by the multiple-choice method. Journal of Animal Behavior, $1915,5,185-225$.

Ierkes, Robert $M$ The mental life of monkeys and apes: a study of ideational behavior. Behavior Monographs, 1916. 3, whole number 12 . 
oratory. Outbred females $\mathrm{A}$ and $\mathrm{B}$ and male $\mathrm{D}$, all of the same litter, were born February 11, 1915, and were about five months old when they were first used, early in July. Inbred male J was born March 1, and began work early in August when five months of age. Inbred female $\mathrm{C}$ from another line was younger than the others, being born March 27 and beginning work when about three months of age.

These fire subjects were selected from nine rats which were given prelimmary training They proved readier in forming the food-association and in getting accustomed to the apparatus than the other four, and it therefore seemed possible to bring them to a more unform state of preparation prior to the actual experiments.

There were, however, very noticeable individual differences from the outset A was rather timid, entering the compartments cautiously and with hesitation. D worked rather slowly and appeared in poor health from the start $B$ and $C$ were extremely active and energetic, choosing successive doors with great rapidity and running to the food-dish at full speed.

The rats were not paired, but two or three of the same sex were caged together.

\section{APPARATUS}

The experiments were performed in a well lighted attic room at the writer's home in Haverhill, Massachusetts. A ground plan of the apparatus is shown in figure 1. It consisted of nine similar compartments, C, C. etc with a door at each end,- - the front door giving access to the reaction-chamber $\mathrm{R}$ and the rear door to the back alley $\mathrm{A}$. The compartments were placed equidistant from the door of the entrance box $\mathrm{E}$, along the arc of a quadrant of a circle.

The various doors, of sheet brass, were arranged to slide vertically and were operated from the experimenter's table by weighted strings passing through screw eyes above the doors. Those doors leading from the reaction-chamber into the nine compartments were arranged to remain 3-16 of an inch from the floor when closed, to avoid pinching the animal's tail. The entire apparatus was covered with wire netting and the floor was sprinkled with a light layer of sawdust.

From the reaction chamber the animal could enter one of the nine compartments, pass into the back alley, thence to the side 
alley and the food-dish F. To facilitate its re-entrance into $\mathrm{E}$, a light wooden door $\mathrm{D}$ was hinged to a frame sliding along the wall of the side alley S. Pulling a string caused this door to swing across the alley and then slide along in a transverse position toward the entrance box. This cut off the animal's retreat and, if necessary, pushed it into the entrance box. Another string pulled this device back to its original position.

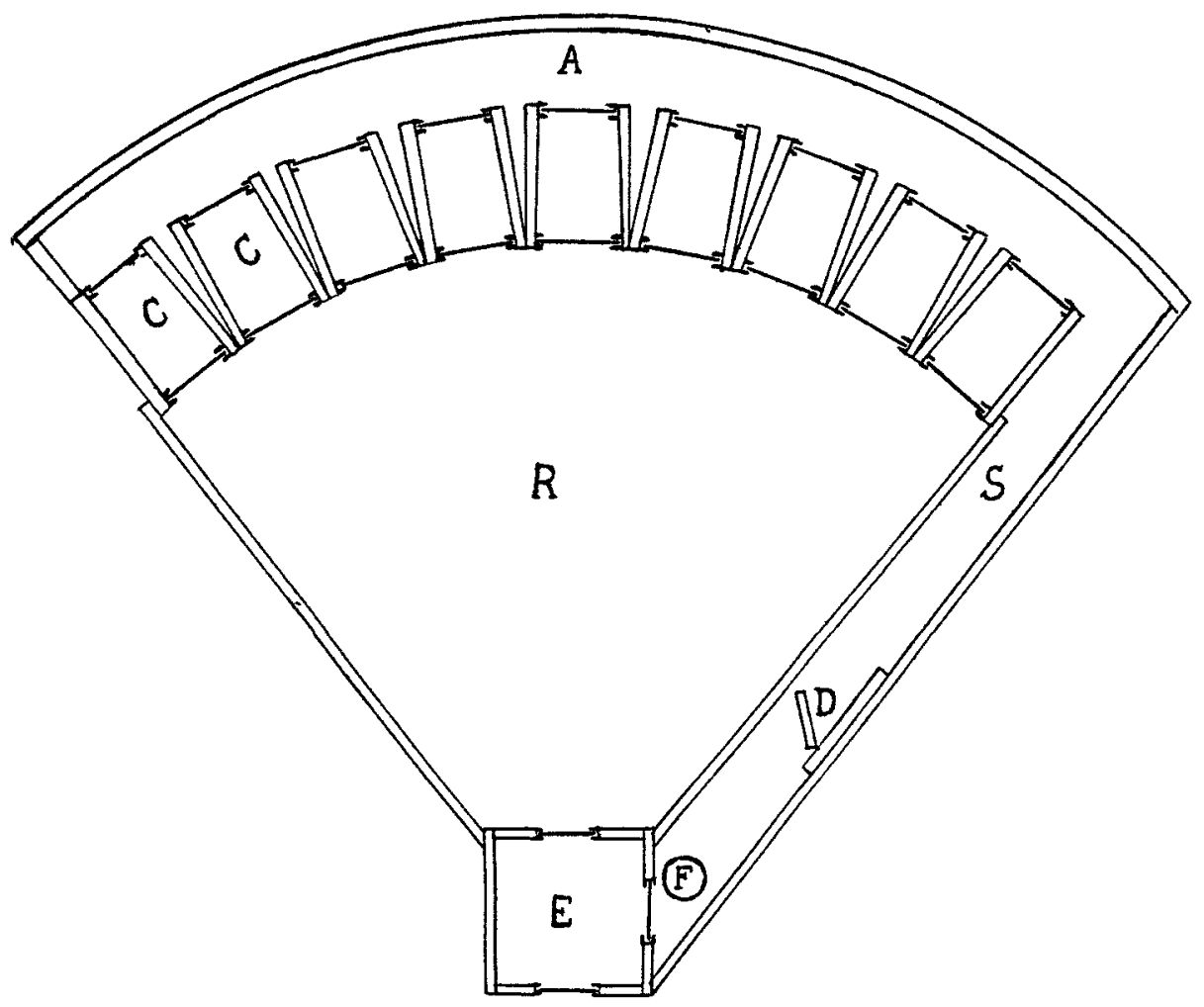

FIGURE 1. Ground plan of multiple-choice apparatus for rats. Scale 1:10

$E$, entrance-box, $R$, reaction-chamber; $C$, reaction-mechanisms; $A$, back alley; $S$, side alley; $D$, sliding door; $F$, food-dish. The doors (except $D$ ) moved vertically and were operated by weighted cords through screw-eyes not shown in the figure.

The interior dimensions of the nine compartments were: width 3 in., length 8 in., depth 4 in. Each front door was 24 in. from the door of the entrance box $\mathrm{E}$. The alleys were $4 \mathrm{in}$. wide, the entrance box $6 \times 6$ in. and the entire apparatus 4 in. deep. 
The apparatus rested on the floor on the side of the room opposite the only window, with the entrance box nearest the window and the front doors of the compartments directed toward the window: The experimenter sat about 10 feet distant behind a table near the window. A large mirror, hung at an angle of 45 degrees from the wall above the apparatus, illuminated it fairly uniformly and enabled the experimenter to observe the animal in positions that could not be seen directly.

\section{PROBLEMS AND GENERAL METHOD}

The first two of the standard problems² were presented to the various subjects, - viz. the selection of the first mechanism on the right and the selection of the second mechanism from the left Below are given the ten settings of the doors which were presented in succession together with the number of the given setting in the series, the number of doors open and the number of the correct door. The doors were numbered from right to left as viewed by the subject.

Problem 1. First mechanism at the right end of the group

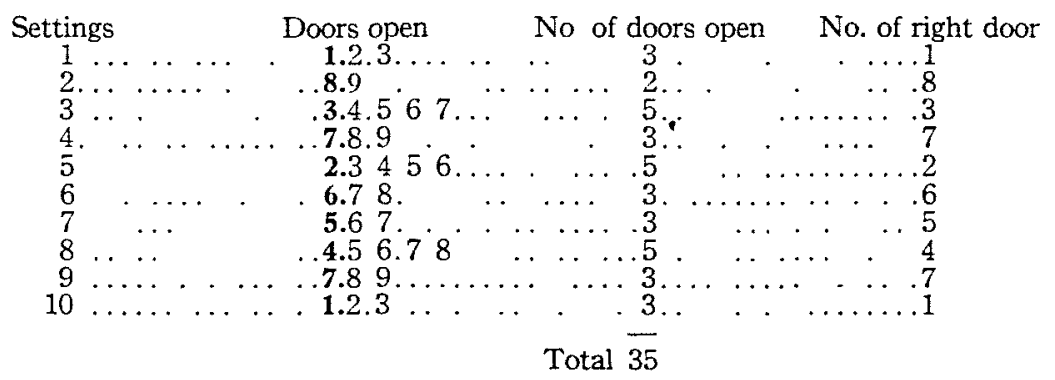

Problem 2. Second mechanism from the left end of the group

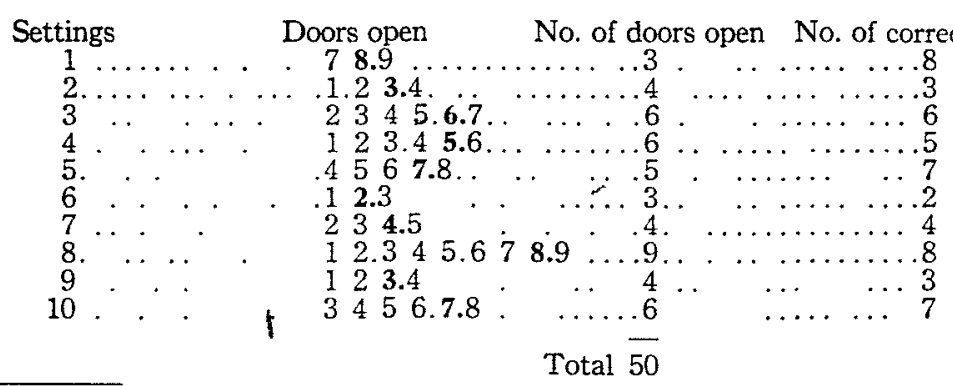

\footnotetext{
${ }^{2} \mathrm{Cl}$. footnote 1 .
} 
In most cases the rats were given the ten settings in order in a single series of experiments. In a few of the earlier series it was not feasible to give ten trials in immediate succession.

Both punishment and reward were used as incentives. The punishment consisted of confinement in a compartment after an incorrect choice for 5 (in some cases 10) seconds. The reward consisted of food,- cold boiled potato or green corn proving most satisfactory The rats were given all the food they wished during the afternoon and evening, but always fasted from $10 \mathrm{p} . \mathrm{m}$ until the middle of the next forenoon when the experimental observations were usually made. If trials were made in the afternoon also, no food was given in the interim.

\section{EXPERIMENTAL PROCEDURE}

The usual procedure in a series was as follows. Sufficient food for 10 trials was placed in small bits in the food-dish. The rat was put into the entrance box through the rear door The experimenter then took his seat at the table ten feet distant and remained there during the entire series The proper entrance doors of the compartments were raised for the first trial, e. g., in Problem 1 doors 1, 2 and 3 were opened and all the others closed. The door leading from $\mathrm{E}$ into the reaction-chamber was then raised and the behavior of the rat was recorded in symbols from its entrance into the chamber until its arrival at the food.

If the animal entered the wrong compartment the door behind 1t was closed for 5 seconds, - the experimenter counting the tics of the stop-watch. The rat was then allowed to retreat from the compartment and make another choice. This procedure was repeated until the rat entered the correct compartment, whereupon the door behind it was closed and the exit-door opened immediately. The rat then ran along the alley to the food-dish. The experimenter immediately pulled a string closing the door across the alley behind the animal. After a few seconds the door from the alley to the entrance box was raised, and the door behind the rat pulled along until it drove or pushed him into the entrance box. Most of the rats, however, soon acquired the habit of picking up a bit of food, waiting until the door in front of them opened and then going into the entrance box to eat at their leisure. Meanwhile the doors were set for the next 
trial and the rat, when ready, was admitted again into the reaction-chamber for another trial. The time from the entrance into the reaction-chamber until the arrival at the food nas measured with a stop-watch.

\section{PRELIMINARY TRAINING}

The rats were placed in the apparatus in groups of two or three with all the doors open and with foot in the food-dish. They were left there for three hours. This procedure was repeated for three days.

Then with all the entrance doors of the nine compartments open, a rat was admitted to the reaction-chamber. When he entered any compartment the door was closed behind him and the exit door immediately rased and he found his way to the food. He was allowed to eat for a few seconds, then pushed into the entrance box and readmitted to the reaction-chamber with all the entrance doors open. This was repeated for half an hour Such preliminary training was continued until the animal made ten choices in half an hour This necessitated in most cases only two or three series, of half hour's length, a day apart

The following day the rat was given the opportunity to secure success at each of the nine boxes. No 1 was the on!y dor open on the first trial, No. 2 on the second, etc. The next day this was reversed, No. 9 being the only door open on the first trial, No. 8 on the second, etc. The rats by this time had the food-association well formed and appeared to be in a state of uniform preparation for the regular experiments

\section{RESULTS OF EXPERIMENTS}

As it is essential to present the data for each trial in the series of experiments, tables I, II, III, IV, V, and VI have been constructed after the following manner. At the head of each table stand the several settings, the letter $\mathrm{S}$ serving as an abbreviation for setting and the number following it designating the place of the setting in the series. Immediately under the number of the setting appear the numbers of the doors open, with the one to be chosen (correct one) in bold face type. The column at the left gives the date. The second column gives the number of the various trials in the whole series. Following these num- 
bers are the results for the trials indicated,-- the figures denoting the numbers of the boxes entered in the order of entrance. The last five columns give the number of correct and incorrect first choices in a given series of ten, the total number of right and wrong first choices on a given day and the daily ratio of right to wrong. At the bottom of the tables for problem 1 appear the results in a check series of ten trials with ten new settings. For example, referring to table I, we see that rat A entered compartment No. 3 on the first trial and after punishment by confinement entered No. 1 which was correct; in the 56th trial she entered No 8 three times in succession. being punished for each entrance and then entered No. 6 the correct one. The time of the trials, although recorded as a matter of routine is not included in the results.

The tables enable one to obtain quickly information concerning the forms of response and the changes therein during the course of the experiment. Further comment will be made in the following section.

\section{DISCUSSION OF RESULTS}

Problem 1, which consisted in the selection of the reaction mechanism on the subject's right, proved soluble for the four rats, i. e., they were able to make correct first choices in ten consecutive trials on the ten settings.

Outbred female A was successful after 170 trials and her brother $\mathrm{D}$ after 200. Inbred male J, of about the same age, solved the problem in 170 trials, while the younger inbred female $\mathrm{C}$ required 350 .

In this problem the total number of doors open in the ten settings is 35 . Of these 10 were of course correct. Hence the probability of a correct first choice in any trial is 10-35 and the probable ratio of correct to incorrect first choices in any series apart from experience is 1 to 2.5 . The tables show that $D$ and $\mathrm{J}$ started with a ratio for the first day of 1 to 9 which is much less efficient than was to be expected, while $A$ and $C$ were more efficient than expectation with ratios of 1 to .66 . These differences were perhaps due to a tendency to go toward the right on the part of $\mathrm{A}$ and $\mathrm{C}$, while with $\mathrm{D}$ the opposite tendency was manifest throughout.

After solution of the problem each animal was given 10 trials 
TABLE I

Rrsults for Rat A, OUtbred Feriale, in Problem 1

\begin{tabular}{|c|c|c|c|c|c|c|c|c|c|c|c|c|c|c|c|c|}
\hline Date & $\begin{array}{l}\text { No. } \\
\text { of } \\
\text { trials }\end{array}$ & $\begin{array}{l}\text { S. } 1 \\
1.2 .3\end{array}$ & $\begin{array}{l}\text { S. } 2 \\
8.9\end{array}$ & $\begin{array}{c}\text { S. } 3 \\
3.45 .67\end{array}$ & $\begin{array}{l}\text { S. } 4 \\
7.8 .9\end{array}$ & $\begin{array}{c}\text { S. } 5 \\
2.3 .4 .5 .6\end{array}$ & $\begin{array}{l}\text { S. } 6 \\
6.7 .8\end{array}$ & $\begin{array}{l}\text { S. } 7 \\
5.67\end{array}$ & $\begin{array}{c}\text { S } 8 \\
4.56 .7 .8\end{array}$ & $\begin{array}{c}5.9 \\
7.8 .9\end{array}$ & $\begin{array}{l}\text { S. } 10 \\
1.2 .3\end{array}$ & $\mathrm{R}$ & W & $\mathrm{R}$ & W & $\begin{array}{l}\text { Ratio } \\
\text { of } \\
\mathrm{R} \text { to } \mathrm{W}\end{array}$ \\
\hline $\begin{array}{c}\text { July } \\
8 \\
9 \\
" \\
10 \\
12 \\
13 \\
14 \\
" \\
15 \\
" \\
16 \\
" \\
17 \\
18 \\
19 \\
4 \\
20\end{array}$ & $\begin{array}{r}1-10 \\
11-20 \\
21-30 \\
31-40 \\
41-50 \\
51-60 \\
61-70 \\
71-80 \\
81-90 \\
91-100 \\
101-110 \\
111-120 \\
121-130 \\
131-140 \\
141-150 \\
151-160 \\
161-170\end{array}$ & $\begin{array}{lll}3.1 & \\
3 & 2 & 1 \\
1 & & \\
2 . & 3 & 1 \\
2 . & 1 & \\
2 & 1 & \\
1 & & \\
2 & 3.2 & 1 \\
3 & 1 & \\
1 & & \\
1 & & \\
2 & 1 & \\
2 & 1 & \\
1 & & \\
2 & 1 & \\
3 & 1 & \\
1 & & \end{array}$ & $\begin{array}{l}8 \\
8 \\
8 \\
8 \\
8 \\
8 \\
8 \\
9 \\
8 \\
8 \\
8 \\
8 \\
8 \\
8 \\
8 \\
8 \\
8\end{array}$ & $\begin{array}{lll}3 & & \\
4 & 7 & 3 \\
3 & & \\
4 & 7 & 3 \\
3 & & \\
6 & 3 \\
5 & 3 \\
4 & 3 \\
3 & \\
5 & 3 \\
3 & \\
3 & & \\
6 & 4.3 \\
3 & \\
3 & \\
7.3 \\
3\end{array}$ & $\begin{array}{ll}8.7 \\
7 \\
7 \\
7 \\
8 & 9 \\
9 & 7 \\
8 & 7 \\
7 & \\
7 & \\
8 & 7 \\
7 & \\
7 \\
7 \\
7 \\
7 \\
9.7 \\
7\end{array}$ & 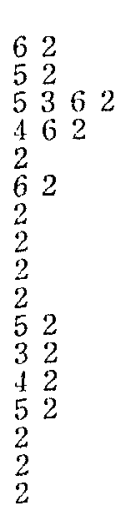 & $\begin{array}{llll}6 & & & \\
7 & 6 & \\
6 & & \\
6 & & & \\
8 & 6 & & \\
8 & 8 & 8 & 6 \\
6 & & & \\
8 & 6 & \\
8 & 6 & \\
6 & & \\
6 & & \\
6 & & \\
6 & & \\
6 & & \\
6 & & \\
8 & 6 \\
6 & & \end{array}$ & $\begin{array}{l}5 \\
675 \\
5 \\
5 \\
65 \\
5 \\
5 \\
5 \\
5 \\
65 \\
5 \\
5 \\
6 \\
5 \\
5 \\
5 \\
5 \\
5\end{array}$ & $\begin{array}{lll}4 & & \\
4 & & \\
4 & & \\
4 & & \\
8 & 4 & \\
6 & 7 & 4 \\
4 & & \\
4 & & \\
4 & & \\
8 & 5 & 4 \\
5 & 4 & \\
4 & & \\
4 & & \\
5 & 7 & 4 \\
5 & 4 & \\
4 & & \\
4 & & \end{array}$ & $\begin{array}{l}7 \\
7 \\
7 \\
7 \\
7 \\
7 \\
9 \\
7 \\
7 \\
9 \\
7 \\
7 \\
7 \\
7 \\
7 \\
7 \\
7 \\
7 \\
7\end{array}$ & 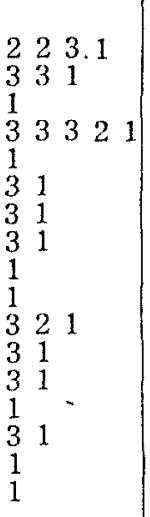 & $\begin{array}{c}6 \\
4 \\
9 \\
6 \\
5 \\
3 \\
6 \\
5 \\
7 \\
6 \\
7 \\
7 \\
5 \\
8 \\
7 \\
6 \\
10\end{array}$ & $\begin{array}{l}4 \\
6 \\
1 \\
4 \\
5 \\
7 \\
4 \\
5 \\
3 \\
4 \\
3 \\
3 \\
5 \\
2 \\
3 \\
4 \\
0\end{array}$ & $\begin{array}{c}6 \\
\\
13 \\
6 \\
5 \\
3 \\
\\
11 \\
\\
13 \\
\\
14 \\
5 \\
8 \\
\\
13 \\
10\end{array}$ & $\begin{array}{l}4 \\
7 \\
4 \\
5 \\
7 \\
9 \\
\\
7 \\
\\
6 \\
5 \\
2 \\
7 \\
7 \\
0\end{array}$ & $\begin{array}{l}1: 0.66 \\
1: 0.54 \\
1: 0.66 \\
11.00 \\
1: 233 \\
1: 081 \\
1: 0.54 \\
1.0 .43 \\
1: 1.00 \\
1: 0.25 \\
1: 0.54 \\
1: 0.00\end{array}$ \\
\hline & & 2.3 .4 & 6.789 & 3.1 .5 & 4.56789 & 6.7 .8 .9 & 1.2 .345 & $\begin{array}{c}2.3 .4 .5 \\
6.7 .8\end{array}$ & 3.4 .5 .6 .7 .8 & 5.6 .7 & $\begin{array}{c}1.2 .3 .4 .5 \\
67.8 .9\end{array}$ & & & & & \\
\hline 20 & $1-10$ & 2 & 6 & 3 & 91 & 6 & 1 & 2 & 3 & 5 & 1 & 9 & 1 & 9 & 1 & $1: 0.11$ \\
\hline $\begin{array}{l}\text { Reten- } \\
\text { tion } \\
\text { Sept. } 9\end{array}$ & & 1 & 8 & 3 & 7 & 2 & 86 & 5 & 4 & 99.7 & 1 & 8 & 2 & & & \\
\hline
\end{tabular}

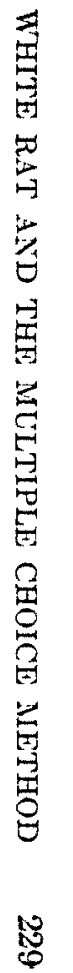


TABLE II

Results for Rat D, OUtbred Male, in Problem I

\begin{tabular}{|c|c|c|c|c|c|c|c|c|c|c|c|c|c|c|c|c|}
\hline Date & $\begin{array}{l}\text { No. } \\
\text { of } \\
\text { trials }\end{array}$ & $\begin{array}{l}\text { S. } 1 \\
1.23\end{array}$ & $\begin{array}{l}\text { S. } 2 \\
8.9\end{array}$ & $\begin{array}{c}\text { S } 3 \\
\mathbf{3 . 4} 56.7\end{array}$ & $\begin{array}{l}\text { S. } 4 \\
7.8 .9\end{array}$ & $\begin{array}{c}\text { S. } 5 \\
2.3 .456\end{array}$ & $\begin{array}{l}\text { S. } 6 \\
6.7 .8\end{array}$ & $\begin{array}{c}\text { S } 7 \\
5.6 .7\end{array}$ & $\begin{array}{c}\text { S. } 8 \\
4.5 .6 .78\end{array}$ & $\begin{array}{c}\text { S. } 9 \\
7.8 .9\end{array}$ & $\begin{array}{l}\text { S. } 10 \\
1.23\end{array}$ & $\mathrm{R}$ & W & $\mathrm{R}$ & W & $\begin{array}{l}\text { Ratio } \\
\text { of } \\
R \text { to } W\end{array}$ \\
\hline \multirow[t]{2}{*}{$\begin{array}{c}\text { July } \\
4 \\
6 \\
7 \\
8 \\
9 \\
12 \\
13 \\
14 \\
1 \\
15 \\
16 \\
16 \\
17 \\
18 \\
19 \\
4 \\
20 \\
4 \\
21\end{array}$} & $\begin{array}{r}1-10 \\
11-20 \\
21-30 \\
31-40 \\
41-50 \\
51-60 \\
61-70 \\
71-80 \\
81-90 \\
91-100 \\
101-110 \\
111-120 \\
121-130 \\
131-140 \\
141-150 \\
151-160 \\
161-170 \\
171-180 \\
181-190 \\
191-200\end{array}$ & $\begin{array}{lll}3 & 2 & 1 \\
1 & & \\
3 & 1 \\
3 & 1 & \\
3 & 1 & \\
3 & 1 \\
3 & 1 & \\
3 & 1 & \\
3 & 1 & \\
3 & 1 & \\
3 & 1 & \\
3 & 2 & 1 \\
3 & 1 & \\
2 & 1 & \\
1 & & \\
1 & & \\
1 & & \\
1 & & \\
1 & & \\
1 & \end{array}$ & $\begin{array}{lll}9 & 8 \\
9 & 9 & 8 \\
9.8 & 8 \\
8 & \\
9 & 8 \\
9 & 8 \\
8 & \\
9 & 8 \\
9 & 8 \\
8 & \\
8 & & \\
9 & 8 \\
8 & \\
9 & 8 \\
8 & \\
8 & \\
8 & \\
8 & \\
8 & \\
8 & \end{array}$ & 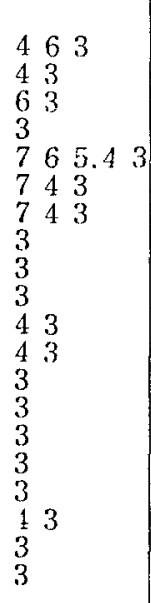 & $\begin{array}{llll}9 & 9 & 8 & 7 \\
9 & 7 & & \\
7 & & \\
8 & 7 & \\
9 & 7 & & \\
9 & 8 & 7 \\
7 & & \\
9 & 7 & \\
7 & & \\
8 & 7 & \\
8 & 7 \\
9 & 7 \\
8 & 7 \\
8 & 7 & \\
8 & 7 \\
7 & & \\
9 & 8.7 \\
9 & 7 \\
8 & 7 \\
7 & & \end{array}$ & 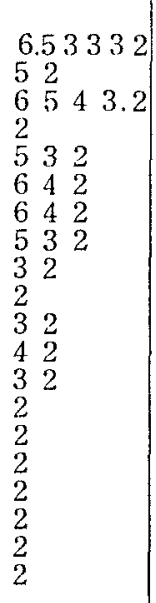 & $\begin{array}{llll}7 & 7 & 7 & 6 \\
6 & & \\
7 & 6 & \\
8 & 6 & \\
8 & 6 & \\
7 & 6 & \\
6 & & \\
8 & 6 & \\
8 & 6 \\
6 & \\
6 & \\
6 & \\
6 & \\
6 & \\
6 & \\
6 & \\
6 & \\
6 & \\
6 & \\
6 & \end{array}$ & 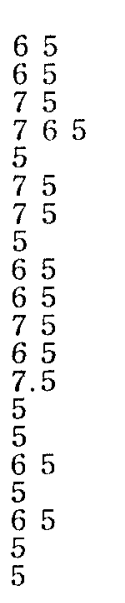 & $\begin{array}{llll}6 & 5 & 4 \\
8 & 7 & 5 & 4 \\
7 & 5 & 4 & \\
8 & 7 & 5 & 4 \\
7 & 6 & 4 & \\
4 & & & \\
4 & & & \\
4 & & & \\
5 & 4 & & \\
5 & 4 & & \\
4 & & & \\
4 & & & \\
8 & 4 & & \\
4 & & & \\
8 & 7 & 6 & 4 \\
4 & & \\
4 & & \\
4 & & & \\
4 & & & \\
4 & & & \end{array}$ & $\begin{array}{l}7 \\
7 \\
7 \\
97 \\
97 \\
97 \\
97 \\
87 \\
77 \\
87 \\
77 \\
7 \\
97 \\
87 \\
77 \\
7 \\
87 \\
87 \\
7 \\
7\end{array}$ & $\begin{array}{lll}3 & 2 & 1 \\
2 & 1 & \\
3 & 2 & 1 \\
3 & 2 & 1 \\
3 & 2 & 1 \\
3 & 1 & \\
3 & 1 & \\
3 & 1 & \\
3 & 1 & \\
3 & 1 & \\
2 & 1 & \\
3 & 1 & \\
3 & 1 & \\
1 & & \\
1 & & \\
1 & & \\
1 & & \\
1 & & \\
3 & 2 & 1 \\
1 & & \end{array}$ & $\begin{array}{l}1 \\
3 \\
2 \\
3 \\
1 \\
1 \\
4 \\
3 \\
3 \\
4 \\
4 \\
3 \\
3 \\
6 \\
8 \\
9 \\
8 \\
6 \\
8 \\
10\end{array}$ & $\begin{array}{l}9 \\
7 \\
8 \\
7 \\
9 \\
9 \\
6 \\
7 \\
7 \\
6 \\
6 \\
7 \\
7 \\
4 \\
2 \\
1 \\
2 \\
4 \\
2 \\
0\end{array}$ & $\begin{array}{l}1 \\
3 \\
2 \\
3 \\
1 \\
1 \\
4 \\
6 \\
8 \\
8 \\
6 \\
6 \\
8 \\
17 \\
14 \\
10\end{array}$ & \begin{tabular}{|c}
9 \\
7 \\
8 \\
7 \\
9 \\
9 \\
6 \\
14 \\
12 \\
12 \\
14 \\
4 \\
2 \\
3
\end{tabular} & $\begin{array}{l}1: 9.00 \\
1: 2.33 \\
1: 4.00 \\
1.2 .33 \\
1: 9.00 \\
1: 9.00 \\
1: 1.50 \\
1: 2.33 \\
1: 1.50 \\
1: 2.33 \\
1: 066 \\
1.0 .25 \\
1: 0.17 \\
1: 0.43 \\
1: 0.00\end{array}$ \\
\hline & & 2.3 .4 & 6.789 & 3.4 .5 & 4.5.6.7.8.9 & 6.7 .8 .9 & $\mathbf{1 . 2 3 4 . 5}$ & $\begin{array}{c}2.3 .4 .5 \\
6.7 .8\end{array}$ & 3.4 .5 .6 .7 .8 & 5.6 .7 & $\begin{array}{c}1.2 .3 .4 .5 \\
67.8 .9\end{array}$ & & & & & \\
\hline 21 & $1-10$ & 2 & 6 & 3 & 4 & 6 & 1 & 2 & 6.43 & 5 & 1 & 9 & 1 & 9 & 1 & 1.0 .11 \\
\hline
\end{tabular}


TABLE III

Results for Rat J, Inbred Male, in Problem 1

\begin{tabular}{|c|c|c|c|c|c|c|c|c|c|c|c|c|c|c|c|c|}
\hline Date & $\begin{array}{c}\text { No. } \\
\text { of } \\
\text { trials }\end{array}$ & $\begin{array}{l}\text { S. } 1 \\
1.23\end{array}$ & $\begin{array}{l}\text { S. } 2 \\
8.9\end{array}$ & $\begin{array}{c}\text { S. } 3 \\
3.4567\end{array}$ & $\begin{array}{l}\text { S } 1 \\
7.8 .9\end{array}$ & $\begin{array}{c}\text { S. } 5 \\
2.3 \pm 5.6\end{array}$ & $\begin{array}{l}\text { S } 6 \\
6.7 .8\end{array}$ & $\begin{array}{l}5.7 \\
5.6 .7\end{array}$ & $\begin{array}{c}\text { S } 8 \\
4.5 .678\end{array}$ & $\begin{array}{r}5.9 \\
7.89\end{array}$ & $\begin{array}{l}\text { S. } 10 \\
1.2 .3\end{array}$ & $\mathrm{R}$ & W & $R$ & W & $\begin{array}{l}\text { Ratio } \\
\text { of } \\
R \text { to } W\end{array}$ \\
\hline \multirow[t]{2}{*}{$\begin{array}{c}\text { Aug. } \\
10 \\
11 \\
1 \\
12 \\
4 \\
13 \\
" \\
11 \\
16 \\
17 \\
18 \\
19 \\
20 \\
" \\
" \\
"\end{array}$} & $\begin{array}{r}1-10 \\
11-20 \\
21-30 \\
31-40 \\
41-50 \\
51-60 \\
61-70 \\
71-80 \\
81-90 \\
91-100 \\
101-110 \\
111-120 \\
121-130 \\
131-140 \\
141-150 \\
151-160 \\
161-170\end{array}$ & $\begin{array}{llll}3 & 2 & 1 \\
3 & 2 & 1 \\
2 & 3 & 1 \\
2 & 3 & 2 & 1 \\
2 & 3 & 2 & 1 \\
3 & 2 & 1 \\
2 & 1 & \\
1 & & \\
1 & & \\
1 & & \\
2 & 1 & \\
3 & 1 & \\
1 & & \\
1 & & \\
2 & 1 & \\
1 & & \\
1 & & \end{array}$ & $\begin{array}{l}8 \\
8 \\
8 \\
8 \\
8 \\
8 \\
8 \\
8 \\
8 \\
8 \\
8 \\
8 \\
8 \\
8 \\
8 \\
8 \\
8\end{array}$ & $\begin{array}{llll}7 & 6 & 5 & 3 \\
7 & 6 & 3 & \\
7 & 7 & 5 & 3 \\
3 & & & \\
3 & & & \\
7 & 4 & 3 \\
4 & 3 & & \\
3 & & & \\
4 & 7 & 3 \\
4 & 3 & \\
4 & 3 & \\
4 & 3 & & \\
4 & 3 & & \\
3 & 3 & & \\
4 & 3 & & \\
4 & 3 & & \\
3 & & & \end{array}$ & $\begin{array}{llll}8 & 9 & 8 & 7 \\
8 & 7 \\
8 & 7 & \\
8 & 7 & \\
7 & & \\
7 & & \\
7 & & \\
7 & & \\
8 & 7 & \\
7 & & \\
7 & & \\
7 & & \\
7 & & \\
7 & & & \\
7 & & & \\
7 & & & \\
7 & & \end{array}$ & $\begin{array}{lll}6 & 3 & 2 \\
6 & 6 & 2 \\
6 & 1 & 2 \\
2 & 2 \\
4 & 3 & 2 \\
2 & & \\
5 & 3 & 2 \\
4 & 3 & 2 \\
2 & \\
3 & 2 \\
4 & 2 \\
4 & 3 & 2 \\
3 & 2 & \\
3 & 6 & 2 \\
3 & 2 \\
3 & 2 \\
2 & 2\end{array}$ & $\begin{array}{llll}8 & 6 & & \\
7 & 8 & 7 & 6 \\
8 & 6 & & \\
7 & 6 & \\
8 & 7 & 6 \\
6 & & \\
6 & & \\
6 & & \\
6 & & \\
6 & & \\
6 & & \\
7 & 8 & 6 \\
6 & & \\
6 & & \\
7 & 6 \\
6 & & \\
6 & & \end{array}$ & $\begin{array}{ll}7 & 5 \\
7 & 5 \\
5 & \\
5 \\
5 \\
5 \\
5 \\
6 & 5 \\
6 & 5 \\
5 & 5 \\
6 & 5 \\
5 & \\
6 & 75 \\
6 & 5 \\
5 & 5 \\
5 & \\
5 & \end{array}$ & $\begin{array}{lll}8 & 6 & 4 \\
5 & 4 & \\
8 & 6 & 4 \\
4 & & \\
4 & \\
4 & & \\
5 & 4 \\
6 & 8 & 4 \\
4 & & \\
4 & \\
4 & \\
4 & \\
4 & & \\
5 & 4 \\
4 & & \\
4 & & \\
4 & & \end{array}$ & $\begin{array}{l}97 \\
8 \\
7 \\
7 \\
7 \\
7 \\
7 \\
7 \\
7 \\
7 \\
7 \\
7 \\
7 \\
7 \\
7 \\
7 \\
7 \\
7\end{array}$ & 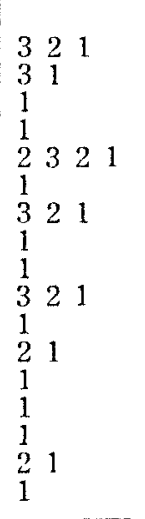 & $\begin{array}{l}1 \\
1 \\
4 \\
7 \\
6 \\
8 \\
5 \\
7 \\
7 \\
7 \\
6 \\
5 \\
7 \\
7 \\
6 \\
7 \\
10\end{array}$ & $\begin{array}{l}9 \\
9 \\
6 \\
3 \\
4 \\
2 \\
5 \\
3 \\
3 \\
3 \\
4 \\
5 \\
3 \\
3 \\
4 \\
3 \\
0\end{array}$ & $\begin{array}{c}1 \\
5 \\
13 \\
\\
13 \\
7 \\
7 \\
\\
13 \\
5 \\
7\end{array}$ & $\begin{array}{c}9 \\
15 \\
7 \\
7 \\
7 \\
3 \\
3 \\
7 \\
5 \\
3\end{array}$ & $\begin{array}{l}1: 9.00 \\
1: 3.00 \\
1.0 .54 \\
10.54 \\
1: 0.43 \\
1: 0.43 \\
1: 0.54 \\
1: 1.00 \\
1: 0.43\end{array}$ \\
\hline & & 2.31 & 6.7 .89 & $\mathbf{3 . 4 . 5}$ & $\mathbf{4 . 5 6 . 7 . 8 9}$ & 6.789 & 1.2 .3 .4 .5 & $\begin{array}{c}2.34 .5 \\
6.78\end{array}$ & 3.456 .7 .8 & 5.6.7 & $\begin{array}{c}1.2 .3 .4 .5 \\
678.9\end{array}$ & & & & & \\
\hline 20 & $1-10$ & 2 & 6 & 3 & 64 & 6 & 1 & 2 & 3 & 5 & 98.5 .32 .1 & 8 & 2 & 8 & 2 & $1: 0.25$ \\
\hline
\end{tabular}


TABLE IV

Results for Rat C, Inbred Female, in Problem 1

\begin{tabular}{|c|c|c|c|c|c|c|c|c|c|c|c|c|c|c|c|c|}
\hline Date & $\begin{array}{c}\text { No. } \\
\text { of } \\
\text { trials }\end{array}$ & $\begin{array}{l}\text { S. } 1 \\
1.2 .3\end{array}$ & $\begin{array}{l}\text { S. } 2 \\
8.9\end{array}$ & $\begin{array}{c}\text { S. } 3 \\
3.4 .5 .6 .7\end{array}$ & $\begin{array}{l}\text { S. } 4 \\
7.8 .9\end{array}$ & $\begin{array}{c}\text { S. } 5 \\
2.34 .5 .6\end{array}$ & $\begin{array}{c}\text { S. } 6 \\
6.7 .8\end{array}$ & $\begin{array}{c}\text { S. } 7 \\
\mathbf{5 . 6 . 7}\end{array}$ & $\begin{array}{c}\text { S. } 8 \\
4.5 .6 .7 .8\end{array}$ & $\begin{array}{c}\text { S. } 9 \\
7.89\end{array}$ & $\begin{array}{l}\text { S. } 10 \\
1.2 .3\end{array}$ & $\mathrm{R}$ & W & $\mathrm{R}$ & $\mathrm{W}$ & $\begin{array}{c}\text { Ratio } \\
\text { of } \\
\text { R to } W\end{array}$ \\
\hline 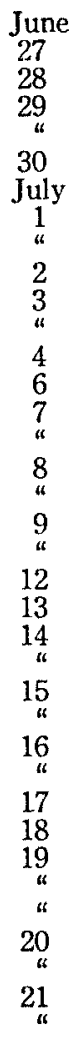 & $\begin{array}{r}51-60 \\
61-70 \\
71-80 \\
81-90 \\
91-100 \\
101-110 \\
111-120 \\
121-130 \\
131-140 \\
141-150 \\
151-160 \\
161-170 \\
171-180 \\
181-190 \\
191-200 \\
201-210 \\
211-220 \\
221-230 \\
231-240 \\
241-250 \\
251-260 \\
261-270 \\
271-280 \\
281-290 \\
291-300 \\
301-310 \\
311-320 \\
321-330 \\
331-340 \\
341-350\end{array}$ & $\begin{array}{lll}3 & 2 & 1 \\
3 & 3 & 1 \\
2 & 1 & \\
2 & 1 & \\
3 & 2 & 1 \\
& & \\
2 & 1 & \\
3 & 2 & 1 \\
2 & 1 & \\
3 & 2 & 1 \\
3 & 2 & 1 \\
3 & 2 & 1 \\
2 & 1 & \\
3 & 1 & \\
2 & 1 & \\
2 & 1 & \\
3 & 2 & 1 \\
3 & 2 & 1 \\
2 & 1 & \\
1 & & \\
2 & 1 & \\
1 & & \\
1 & & \\
2 & 1 & \\
1 & & \\
1 & & \\
1 & & \\
2 & 1 \\
1 & & \\
1 & & \\
1 & & \\
1 & & \\
1 & & \\
1 & & \\
1 & & \\
1 & & \end{array}$ & $\begin{array}{l}8 \\
8 \\
8 \\
8 \\
8 \\
\\
8 \\
9 \\
8 \\
8 \\
8 \\
8 \\
8 \\
8 \\
8 \\
8 \\
8 \\
8 \\
8 \\
8 \\
8 \\
8 \\
8 \\
8 \\
8 \\
8 \\
8 \\
8 \\
8 \\
8 \\
8 \\
8 \\
8 \\
8 \\
8 \\
8 \\
8\end{array}$ & 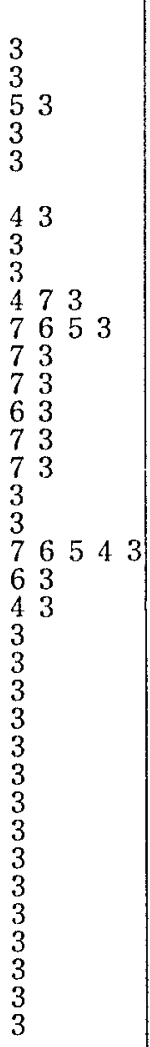 & $\begin{array}{l}7 \\
7 \\
7 \\
7 \\
7 \\
87 \\
7 \\
7 \\
7 \\
7 \\
7 \\
7 \\
7 \\
7 \\
7 \\
7 \\
7 \\
89 \\
7 \\
7 \\
7 \\
7 \\
7 \\
7 \\
7 \\
87 \\
7 \\
7 \\
7 \\
7 \\
7 \\
7 \\
7 \\
7 \\
7\end{array}$ & 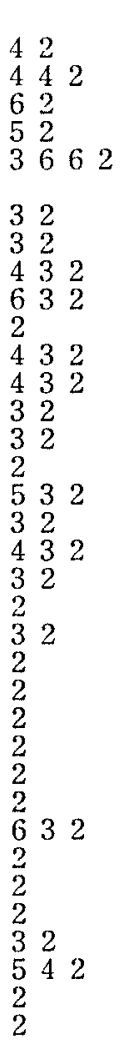 & $\begin{array}{ll}6 & \\
6 & \\
6 & \\
7 & 6 \\
8 & 6 \\
& \\
6 & \\
6 & \\
8 & 6 \\
7 & 6 \\
6 & \\
6 & \\
7 & 6 \\
7 & 6 \\
6 & \\
6 & \\
6 & \\
6 & \\
6 & \\
8 & 7 \\
7 & 6 \\
6 & \\
7 & 6 \\
7 & 6 \\
6 & \\
6 & \\
6 & \\
6 & \\
6 & \\
6 & \\
7 & 6 \\
6 & \\
6 & \\
6 & \\
6 & \\
6 & \end{array}$ & $\begin{array}{ll}7 & 5 \\
7 & 5 \\
5 & \\
5 & \\
7 & 5 \\
& \\
7 & 6 \\
5 & 5 \\
5 & \\
6 & 5 \\
5 & \\
7 & 5 \\
6 & 5 \\
7 & 5 \\
7 & 5 \\
5 & \\
5 & \\
5 & \\
6 & 5 \\
6 & 5 \\
5 & \\
6 & 5 \\
6 & 5 \\
5 & \\
6 & 5 \\
6 & 5 \\
6 & 5 \\
7 & 5 \\
6 & 5 \\
7 & 5 \\
5 & \\
5 & \\
5 & 5 \\
7 & 5 \\
5 & \end{array}$ & 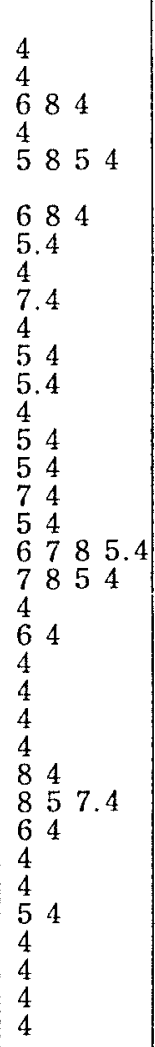 & $\begin{array}{l}7 \\
7 \\
9 \\
7 \\
7 \\
\\
9 \\
7 \\
7 \\
9 \\
7 \\
7 \\
7 \\
7 \\
9.7 \\
7 \\
7 \\
7 \\
7 \\
87 \\
7 \\
7 \\
7 \\
7 \\
7 \\
7 \\
7 \\
7 \\
7 \\
7 \\
7 \\
7 \\
899 \\
7 \\
7 \\
7 \\
7\end{array}$ & $\begin{array}{lll}3 & 1 \\
3 & 1 \\
3 & 1 & \\
3 & 2 & 1 \\
2 & 1 & \\
& & \\
1 & & \\
2 & 1 & \\
3 & 3 & 1 \\
3 & 2.2 .1 \\
1 & & \\
3 & 2 & 1 \\
1 & & \\
2 & 1 & \\
3 & 2 & 1 \\
3 & 2 & 1 \\
2 & 1 & \\
2 & 1 & \\
3 & 2 & 1 \\
1 & & \\
2 & 1 \\
1 & \\
1 & & \\
2 & 1 \\
1 & \\
2 & 1 \\
1 & \\
1 & & \\
2 & 3.1 \\
1 & & \\
1 & \\
1 & \\
1 & \\
1 & \\
1 & \\
1 & \end{array}$ & $\begin{array}{l}6 \\
6 \\
4 \\
6 \\
4 \\
\\
3 \\
5 \\
5 \\
3 \\
8 \\
4 \\
4 \\
3 \\
4 \\
6 \\
6 \\
6 \\
2 \\
5 \\
6 \\
7 \\
8 \\
7 \\
9 \\
8 \\
7 \\
7 \\
6 \\
9 \\
9 \\
8 \\
9 \\
9 \\
9 \\
10\end{array}$ & $\begin{array}{l}4 \\
4 \\
6 \\
4 \\
6 \\
7 \\
5 \\
5 \\
7 \\
2 \\
6 \\
6 \\
7 \\
6 \\
4 \\
4 \\
4 \\
8 \\
5 \\
4 \\
3 \\
2 \\
3 \\
1 \\
2 \\
3 \\
3 \\
4 \\
1 \\
1 \\
2 \\
1 \\
1 \\
1 \\
0\end{array}$ & $\begin{array}{c}15 \\
16 \\
15 \\
7 \\
6\end{array}$ & $\begin{array}{l}12 \\
5 \\
4\end{array}$ & $\begin{array}{l}1: 150 \\
1: 1.00 \\
1 \cdot 0.81 \\
1: 1.50 \\
1: 1.50 \\
1 \cdot 1.85 \\
1: 0.66 \\
1.1 .50 \\
1: 100 \\
1: 0.66 \\
1: 033 \\
1: 0.25 \\
1: 033 \\
1: 043 \\
1: 0.66\end{array}$ \\
\hline & & 2.3 .4 & 6.7 .8 .9 & 3.4 .5 & 4.5.6.7.8 & 6.7 .8 .9 & 1.2 .3 .4 .5 & $\begin{array}{c}2.3 .4 .5 \\
6.7 .8\end{array}$ & 3.4.5.6.7.8 & 5.6 .7 & $\begin{array}{c}1.2 .3 .4 .5 \\
6.7 .8 .9\end{array}$ & & & & & \\
\hline 22 & $1-10$ & 2 & 6 & 3 & 4 & 6 & 1 & 2 & 3 & 5 & 1 & 10 & 0 & 10 & 0 & $1: 0.00$ \\
\hline
\end{tabular}


on 10 new settings to check the possibility of memorizing the particular settings rather than actually solving the problem. One of the rats did the check series perfectly, two others with only one mistake, and the other with two mistakes. It is evident that all formed the habit of selecting the compartment at the extreme right.

A number of interesting individual tendencies were manifested in the course of the experment. A's method of reaction was usually as follows: passing down the right wall of the reactionchamber to compartment No. 1, she turned leftward and followed along close to the compartments until she reached the first open door. In many instances she turned from the right wall before reaching compartment No. 1 , with the result that she entered a door too far to the left. This factor was of course more operative with settings comprising doors at the right end. It is evident from table I that settings $1,3,5$ and 10 yield a larger number of incorrect first choices than do the others. With settings farther toward the left the first approach to the doors brought the sizject more often in front of a closed door and hence the first open one encountered was correct. On Sept. 9, over seven weeks after the discontinuance of A's training, she was given 10 trials with the original settings and made 8 correct choices out of 10 . Evidently the habit was still strong.

$D$ manifested a tendency entirely different from that of $\mathrm{A}$. He ran along the left wall of the reaction-chamber and across in front of the open doors until he reached a closed one, whereupon he turned back into the last open one. This turning back appeared to be caused by the touch of the vibrissae on the closed door rather than by the sight of it. The turning was always in the same direction,- leftward or toward the doors. D experienced more difficulty than the other rats with setting No. 2, i. e., doors 8 and 9. Going down the left wall he often entered compartment No. 9, whereas he normally turned at that point. As he did poorly also on the other settings involving No. 9, it would appear that he was reacting by tactual rather than visual criteria,-habitually going along the wall until he encountered an obstacle and then turning across, etc. The directness with which No. 9 was entered in contrast with any other door was noticeable. A month after the training ended, although sick and able to move only slowly, he performed two trials correctly 
on the first two settings, employing his customary method of reaction.

J manifested, like $A$, a tendency to go down the right wall of the reaction chamber to compartment No. 1 and then in front of the closed doors to the first open one. He was often, however, premature in turning to the left thus missing the first open door, and he often lost the latter part of his habit and passed several open doors without entering. As contrasted with $A$, he was less ready in recovery from mistakes. Both solved the problem in 170 trials. but whereas with A there were 17 trials in which more than one incorrect choice was made before the correct one, there were 31 such trials with J. It is to be remembered that $J$ was inbred and A outbred.

$\mathrm{C}$ was extremely rapid in her motions, rushing toward the compartments the moment she was released from the entrance box. In her correct trials the total time from entrance-box, through the compartment and alleys to the food was frequentiy 5 seconds. She often ran along the back alley so rapidly that she slipped and fell at the turn into the side alley. This tendency to hasten was perhaps instrumental in her failure to solve the problem in less than 350 trials. She usually appeared to take her orientation from door No. 1, going thence leftward to the correct door. The settings comprising doors at the left proved easy by this method. The most noticeable thing about her behavior was the suddenness with which she sometimes became thus oriented. Frequently when pausing and looking in the wrong door. or smelling about in almost any part of the reaction-chamber she would suddenly dash to door No. 1 and then across into the correct one It is possible that this is the same phenomenon mentioned by Watson, ${ }^{3}$ where a tramed rat, placed in a part of the maze other than the entrance, runs about at random and suddenly darts off correctly, having passed over a "kinaesthetic unit" which arouses a proper sequence of kinaesthetic impulses.

Problem 2, which is definable as the second mechanism from the left end of the group, proved insoluble for the two rats $\mathrm{C}$ and $B$ in 800 and 900 trials respectively.

For the settings of this problem the total number of open doors is 50 with 10 of course correct. Hence the probability of

Watson, J. B. Behavior. New York, 1914, p. 218. 
TABLE $\mathrm{V}$

Results for Rat C, Inbren Female, in Problem 2

\begin{tabular}{|c|c|c|c|c|c|c|c|c|c|c|c|c|c|c|c|c|}
\hline Date & $\begin{array}{c}\text { No. } \\
\text { of } \\
\text { trials }\end{array}$ & $\begin{array}{l}\text { S. } 1 \\
7.8 .9\end{array}$ & $\begin{array}{c}\text { S. } 2 \\
1.2 .3 .4\end{array}$ & $\begin{array}{c}\text { S. } 3 \\
234.5 .6 .7\end{array}$ & $\begin{array}{c}\text { S. } 4 \\
1.2 .3 .45 .6\end{array}$ & $\begin{array}{c}S 5 \\
45.6 .7 .8\end{array}$ & $\begin{array}{l}\text { S. } 6 \\
1.2 .3\end{array}$ & $\begin{array}{c}\text { S. } 7 \\
2.3 .4 .5\end{array}$ & $\begin{array}{c}\text { S. } 8 \\
1.2 .3 .4 .5 \\
6.78 .9\end{array}$ & $\begin{array}{c}\text { S. } 9 \\
12.3 .4\end{array}$ & $\begin{array}{c}\text { S. } 10 \\
3.4 .5 .67 .8\end{array}$ & $R$ & w & $R$ & W & $\begin{array}{l}\text { Ratio } \\
\text { of } \\
\mathrm{R} \text { to } \mathrm{W}\end{array}$ \\
\hline $\begin{array}{l}\text { July } \\
26\end{array}$ & $1-10$ & $\left\{\begin{array}{llll}7 & 7 & 7 & 7 \\
9 & 7 & 8\end{array}\right.$ & 3 & 22276 & $\left\{\begin{array}{llll}3 & 2 & 1 & 6 \\
1 & 4 & 5\end{array}\right.$ & 44467 & 112 & 24 & +678 & 3 & $\left\{\begin{array}{lll}3 & 3 & 4.5 \\
3 & 6 & 7\end{array}\right.$ & 2 & 8 & 2 & 8 & $1: 4.00$ \\
\hline 27 & $11-20$ & 7.7 .79 .7 .8 & 43 & 52246 & 5 & 467 & $\left|\begin{array}{lllll}1 & 3 & 1 & 1 & 3\end{array}\right|$ & 24 & $1 \begin{array}{llll}1 & 3 & 4 & 5\end{array}$ & 13 & 3457 & 1 & 9 & & & \\
\hline “ & $21-30$ & 7.8 & 123 & 6 & 5 & 67 & 12 & 234 & 5678 & $\left\{\begin{array}{llll}4 & 1 & 2 & 1 \\
2 & 1 & 3\end{array}\right.$ & 67 & 2 & 8 & 3 & 17 & $1: 5.66$ \\
\hline 28 & $31-40$ & 78 & 123 & 234.56 & 6135 & 64567 & $\begin{array}{llll}3 & 1 & 3.3 & 2\end{array}$ & $\left\{\begin{array}{lllll}2 & 5 & 3 & 5 & 3 \\
5 & 0 & 3 & 5 & 4\end{array}\right]$ & 4567.8 & $\begin{array}{lllll}4 & 4 & 1 & 4 & 3\end{array}$ & 4567 & 0 & 10 & & & \\
\hline “ & $41-50$ & 78 & 123 & 23456 & $\begin{array}{llll}6 & 1 & 3 & 5 \\
3 & 2 & 4 & 1 \\
1 & 6.3\end{array} \mid$ & 67 & 133332 & 4 & 45678 & 43 & $\begin{array}{lllll}3 & 4 & 5 & 6 & 7\end{array}$ & 1 & 9 & 1 & 19 & $1: 1900$ \\
\hline 29 & $51-60$ & 78 & 41.3 & 45.6 & $\left\{\begin{array}{l}1.2 .3 .4 \\
32\end{array} \mid 65\right.$ & 567 & 32 & 4 & 5678 & 43 & 4.57 & 1 & 9 & & & \\
\hline$" "$ & $\begin{array}{l}61-70 \\
71-80 \\
81-90\end{array}$ & $\begin{array}{l}7.8 \\
78 \\
7.78\end{array}$ & $\begin{array}{lllll}3 & & & \\
4 & 2.1 & 1 & 3 \\
2 & 3 & & \end{array}$ & 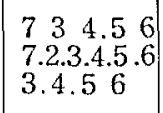 & $\left|\begin{array}{lllll}1 & 3 & 4 & 0 & \\
6 & 2 & 3 & 4 & 5 \\
6 & 2 & 1 & 1\end{array}\right|$ & $\begin{array}{l}4567 \\
787\end{array}$ & $\begin{array}{lll}3 & 3 & 2 \\
3 & 2 \\
1 & 2\end{array}$ & $\begin{array}{lll}2 & 3 & 4 \\
5 & 3 & 4 \\
3 & 4\end{array}$ & $\begin{array}{lllll}4 & 1 & 9 & 9 & 8 \\
6 & 7 & 8 & \\
5 & 6 & 7 & 8\end{array} \mid$ & 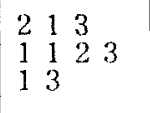 & $\begin{array}{lllll}5 & 7 & & \\
8 & 6 & 7 & & \\
8 & 3 & 5 & 6 & 7\end{array}$ & $\begin{array}{l}1 \\
1 \\
0\end{array}$ & $\begin{array}{c}9 \\
9 \\
10\end{array}$ & 3 & 37 & 1.12 .33 \\
\hline 30 & $\begin{array}{r}91-100 \\
101-110\end{array}$ & 78 & $\begin{array}{lll}12 & 3 \\
1 & 2 & 3\end{array}$ & 76 & $\left|\begin{array}{llll}2 & 3 & 4 & 5 \\
2 & 3 & 4 & 5 \\
4 & 2 & 3 & 4\end{array}\right|$ & 4567 & $\begin{array}{lll}3 & 1 & 2 \\
3 & 2\end{array}$ & $\begin{array}{lll}2 & 3 & 4 \\
2 & 3 & 4\end{array}$ & & $\begin{array}{lll}1 & 2 & 3 \\
1 & 2 & 3\end{array}$ & $\begin{array}{lllll}8 & 4 & 5 & 6 & 7 \\
8 & 4 & 5 & 6 & 7\end{array}$ & 1 & $\begin{array}{l}9 \\
9\end{array}$ & 2 & 18 & 1.900 \\
\hline 31 & $111-120$ & 8 & 123 & $4.566^{3}$ & $\left\{\begin{array}{lllll}1 & 2 & 5 & 4 \\
1 & 2 & 1 & 2 \\
3 & 4 & 5 & 2\end{array} \mid\right.$ & 867 & 32 & 54 & 12.3 .4 .5 .8 & $\begin{array}{lll}1 & 2 & 3 \\
1 & 2\end{array}$ & 96567 & 1 & 9 & 1 & 9 & $1: 9.00$ \\
\hline$\underset{4}{\text { Aug. }}$ & $121-130$ & 7.8 & 3 & 7.456 & 345 & 867 & 1.2 & 234 & 78 & $\left\{\begin{array}{llll}4 & 2 & 1 & 4\end{array}\right.$ & 8567 & 1 & 9 & & & \\
\hline “ & $131-140$ & 8 & 3 & $\left\{\begin{array}{l}3.4 .23 \\
45\end{array}\right.$ & 6.1 .2 .3 .4 .5 & $856 \times 7$ & 32 & 54 & 78 & 43 & 3.84 .5 .6 .7 & 2 & 8 & 3 & 17 & $1: 5.66$ \\
\hline 5 & $141-150$ & 98 & 3 & $\left\{\begin{array}{llll}7 & 2 & 3 & 2 \\
3 & 4 & 4 & 5\end{array}\right.$ & 665 & 84567 & 13.2 & 5234 & 8 & 23 & 7 & 3 & 7 & & & \\
\hline$"$ & $\begin{array}{l}151-160 \\
161-170\end{array}$ & $\begin{array}{ll}9 & 7.8 \\
9 & 78\end{array}$ & $\begin{array}{ll}4 & 3 \\
4 & 2 \\
2 & 3\end{array}$ & $\left|\begin{array}{ccccc}0 & 4 & 5 & 0 \\
2 & 3 & 4 & 5 & 6 \\
2 & 3 & 2 & 4 & 7 \\
3 & 4.5 & 6\end{array}\right|$ & $\left|\begin{array}{cccc}2 & 3 & 4 & 5 \\
6 & 1.2 .3 .4 & .5\end{array}\right|$ & $\begin{array}{llll}7 & 5 & 6 & \end{array}$ & $\begin{array}{ll}12 & 2 \\
3 & 2\end{array}$ & $\begin{array}{l}4 \\
54\end{array}$ & $\begin{array}{ll}78 \\
7 & 8\end{array}$ & $\begin{array}{ll}4 & 3 \\
1 & 2 \\
1\end{array}$ & $\begin{array}{l}7 \\
8.34 .5 .6 .7\end{array}$ & $\begin{array}{l}3 \\
0\end{array}$ & $\begin{array}{c}7 \\
10\end{array}$ & & & \\
\hline
\end{tabular}


TABLE V-Continued

Results for Rat C, Inbred Female, in Problem 2

\begin{tabular}{|c|c|c|c|c|c|c|c|c|c|c|c|c|c|c|c|c|}
\hline Date & $\begin{array}{l}\text { No. } \\
\text { of } \\
\text { trials }\end{array}$ & $\begin{array}{l}\text { S. } 1 \\
7.8 .9\end{array}$ & $\begin{array}{c}\text { S. } 2 \\
12.3 .4\end{array}$ & $\begin{array}{c}\text { S. } 3 \\
2.3 .4 .5 .6 .7\end{array}$ & $\begin{array}{c}\text { S. } 4 \\
1.2 .3 .45 .6\end{array}$ & $\begin{array}{c}\text { S. } 5 \\
4.5 .6 .7 .8\end{array}$ & $\begin{array}{l}\text { S. } 6 \\
12.3\end{array}$ & $\begin{array}{c}S .7 \\
2.3 .4 .5\end{array}$ & $\begin{array}{c}\text { S. } 8 \\
1.2 .3 .4 .5 \\
6.7 .8 .9\end{array}$ & $\begin{array}{c}\text { S. } 9 \\
1.2 .3 .4\end{array}$ & $\begin{array}{c}\$ 10 \\
3.4 .5 .6 .7 .8\end{array}$ & $\mathrm{R}$ & $W$ & $\mathrm{R}$ & W & $\begin{array}{l}\text { Ratio } \\
\text { of } \\
R \text { to } W\end{array}$ \\
\hline Aug. & $171-180$ & 8 & 42123 & 7.76 & $\left\{\begin{array}{llll}4 & 3 & 2 & 1\end{array}\right.$ & 8.867 & 32 & 53.4 & 8 & 423 & 84.567 & 2 & 8 & 8 & 32 & $1: 4.00$ \\
\hline 6 & $181-190$ & 78 & 12.3 & 7456 & $\left\{\begin{array}{lllll}0 & 4 & 3 & & \\
6.3 .2 & .3 & 4 & 6 \\
1 & 2 & 3 & 4 & 5\end{array} \mid\right.$ & 5.67 & 32 & 4 & 78 & 43 & $\left\{\begin{array}{llll}8 & 1 & 3 & 4 \\
5 & 6 & 7\end{array}\right.$ & 1 & 9 & 1 & 9 & $1: 9.00$ \\
\hline 7 & $\begin{array}{l}191-200 \\
201-210\end{array}$ & $\begin{array}{l}8 \\
7.8\end{array}$ & $\begin{array}{llll}2 & 1 & 2 & 3 \\
4 & 3 & & \end{array}$ & $\left\{\begin{array}{llll}6 & & & \\
7 & 7 & 2 & 3 \\
4 & 5 & 6\end{array}\right.$ & $\begin{array}{lllll}4 & 5 & & & \\
6 & 2 & 3 & 4 & 5\end{array}$ & $\begin{array}{l}7 \\
7\end{array}$ & $\begin{array}{ll}2 & \\
1 & 32\end{array}$ & $\begin{array}{ll}5 & 24 \\
5 & 4\end{array}$ & $\begin{array}{ll}78 \\
78\end{array}$ & $\begin{array}{llll}4 & 3 & & \\
2 & 1 & 4 & 2.3\end{array}$ & $\begin{array}{ll}7 & \\
8 & 7\end{array}$ & $\begin{array}{l}5 \\
1\end{array}$ & $\begin{array}{l}5 \\
9\end{array}$ & & & \\
\hline$"$ & $211-220$ & 78 & 123 & 77456 & $\begin{array}{lllll}6 & 2 & 3 & 4 & 5\end{array}$ & $\begin{array}{lllll}8 & 4 & 5 & 6 & 7\end{array}$ & 312 & 34 & 8 & 4123 & 8.7 & 1 & 9 & 7 & 23 & $1: 3.28$ \\
\hline 9 & $221-230$ & 8 & 13 & $\begin{array}{lllll}4 & 57 & 7\end{array}$ & 65 & 84.447 & & 34 & 8 & 13 & 587 & 3 & 7 & 3 & 7 & $1: 2.33$ \\
\hline 10 & $231-240$ & 78 & 423 & 6 & 6.1 .2 .34 .5 & 7 & 32 & 5354 & 8 & +3 & 87 & 3 & 7 & 3 & 7 & $1: 2.33$ \\
\hline $\begin{array}{l}11 \\
12\end{array}$ & $\begin{array}{l}241-250 \\
251-260\end{array}$ & $\begin{array}{l}78 \\
78\end{array}$ & & $\begin{array}{l}4.23 .456 \\
356\end{array}$ & $\begin{array}{l}6.1 .23 .4 .5 \\
65\end{array}$ & $\begin{array}{lllll}8 & 6 & 7 & & \\
8 & 4 & 5 & 6 & 7\end{array}$ & $\begin{array}{lll}1 & 3 & 2 \\
3 & 2\end{array}$ & $\begin{array}{l}4 \\
5\end{array}$ & $\begin{array}{l}8 \\
8\end{array}$ & $\begin{array}{llll}2 & 4 & 1 & 2.3 \\
4 & 3 & & \end{array}$ & $\begin{array}{lllll}8 & 7 & & & \\
8 & 3 & 5 & 6 & 7\end{array}$ & $\begin{array}{l}3 \\
2\end{array}$ & $\begin{array}{l}7 \\
8\end{array}$ & $\begin{array}{l}3 \\
2\end{array}$ & $\begin{array}{l}7 \\
8\end{array}$ & $\begin{array}{l}1: 2.33 \\
1: 4.00\end{array}$ \\
\hline 13 & $261-270$ & 78 & 3 & $\left\{\begin{array}{lllll}4 & 3 & 2 & 7 & 5 \\
4 & 3 & 2 & 7 & 6\end{array} \mid\right.$ & 6.13 .45 & 84567 & 32 & 54 & 8 & 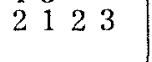 & $\left\{\begin{array}{llll}8 & 8 & 1 & 5 \\
6 & 7\end{array}\right.$ & 2 & 8 & 2 & 8 & $1: 4.00$ \\
\hline 14 & $271-280$ & 78 & 4.3 & 76 & $\left\{\begin{array}{llll}1 & 3 & 2 & 1 \\
6 & 3 & 4 & 5\end{array}\right.$ & 7 & 32 & 54 & 8 & 3 & 87 & 3 & 7 & 3 & 7 & $1: 2.33$ \\
\hline $\begin{array}{l}16 \\
17\end{array}$ & $\begin{array}{l}281-290 \\
291-300\end{array}$ & $\begin{array}{ll}78 \\
78\end{array}$ & $\begin{array}{l}3 \\
43\end{array}$ & $\left\{\begin{array}{rrrrr}7 & 4 & 3 & 7 & 6 \\
7 & 2 & 3 & 7 & \end{array}\right.$ & $\begin{array}{l}10 \\
5 \\
5\end{array}$ & $\begin{array}{lll}8 & 5 & 67 \\
8 & 7\end{array}$ & $\begin{array}{ll}32 \\
32\end{array}$ & $\begin{array}{llll}5 & 2 & 3 & 4 \\
5 & 2 & 5 & 4\end{array}$ & $\begin{array}{l}8 \\
8\end{array}$ & $\begin{array}{lll}2 & 1 & 3 \\
4 & 3\end{array}$ & $\begin{array}{ll}8 & 7 \\
8 & 7\end{array}$ & $\begin{array}{l}3 \\
2\end{array}$ & $\begin{array}{l}7 \\
8\end{array}$ & 3 & 7 & $1: 2.33$ \\
\hline “ & $301-310$ & 8 & 43 & $\left\{\begin{array}{llll}4 & 5 & 0 & \\
7 & 2 & 3 & 4 \\
3 & 4 & 5 & 6\end{array}\right.$ & 6.3 .23 .4 .5 & $\begin{array}{lllll}8 & 5 & 4 & 6.7\end{array}$ & 312 & 54 & 8 & 43 & $\left\{\begin{array}{llll}8 & 6 & 5 & 4 \\
3 & 5 & 6 & 7\end{array}\right.$ & 2 & 8 & 4 & 16 & $1: 4.00$ \\
\hline 18 & $311-320$ & 7.8 & 43 & 2.3.5.4.7.6 & $\left\{\begin{array}{llll}6 & 3 & 4 & 3 \\
2 & 4 & 5\end{array}\right.$ & 87 & 1.2 & 4 & 8 & 3 & 4367 & 3 & 7 & 3 & 7 & $1: 2.33$ \\
\hline 20 & $\begin{array}{l}321-330 \\
331-310\end{array}$ & $\begin{array}{ll}78 \\
78\end{array}$ & $\begin{array}{lllll}2 & 4 & 1 & 2 & 3 \\
2 & 3 & & & \end{array}$ & $\begin{array}{lll}7 & 5 & 6 \\
4 & 5 & 6\end{array}$ & $\begin{array}{llll}6 & 3 & 4 & 5 \\
6 & 5 & \end{array}$ & $\begin{array}{ll}8 & 7 \\
8 & 7\end{array}$ & $\begin{array}{ll}32 \\
32\end{array}$ & $\begin{array}{l}354 \\
4\end{array}$ & $\begin{array}{l}98 \\
5.4 .3 .2 \\
5\end{array}$ & $\begin{array}{lll}1 & 2 & 3 \\
1 & 2 & 3\end{array}$ & $\begin{array}{l}7 \\
87\end{array}$ & $\begin{array}{l}1 \\
1\end{array}$ & $\begin{array}{l}9 \\
9\end{array}$ & 2 & 18 & $1: 9.00$ \\
\hline 21 & $341-350$ & 78 & 3 & 7.245 .4 .6 & $\left\{\begin{array}{llll}6 & 2 & 3 & 2 \\
1 & 4 & 5 & \end{array}\right.$ & 87 & 32 & 34 & 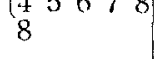 & 2143 & 7 & 3 & 7 & 3 & 7 & $1: 2.33$ \\
\hline 23 & $351--360$ & 78 & 3 & 6 & $\left\{\begin{array}{lll}1 & 4 & 5 \\
3 & 2 & 4 \\
2 & 1 & 5\end{array} 3\right.$ & 87 & 3132 & 54 & 8 & 43 & 87 & 3 & 7 & & & \\
\hline
\end{tabular}




\begin{tabular}{|c|c|c|}
\hline “ & $361-370$ & 7.8 \\
\hline “ & $371-380$ & 7.8 \\
\hline 24 & $381-390$ & 7.8 \\
\hline 25 & $\begin{array}{l}391-400 \\
401-410\end{array}$ & $\begin{array}{l}788 \\
8\end{array}$ \\
\hline " & $\begin{array}{l}411-420 \\
421-430\end{array}$ & $\begin{array}{l}8 \\
78 \\
8\end{array}$ \\
\hline 26 & $431-440$ & 78 \\
\hline 27 & $\begin{array}{l}441-450 \\
451-460\end{array}$ & $\begin{array}{l}78 \\
7.8\end{array}$ \\
\hline 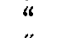 & $461-470$ & 78 \\
\hline “ & $\begin{array}{l}471-480 \\
481-490\end{array}$ & 88 \\
\hline 28 & $491-500$ & 8 \\
\hline 30 & $\begin{array}{l}501-510 \\
511-520\end{array}$ & $\begin{array}{l}78 \\
8\end{array}$ \\
\hline 31 & $521-530$ & 78 \\
\hline “ & $531-540$ & 8 \\
\hline " Sent & $541-550$ & 8 \\
\hline 1 & $551-560$ & 8 \\
\hline 2 & $\begin{array}{l}561-570 \\
571-580\end{array}$ & $\begin{array}{ll}98 \\
8\end{array}$ \\
\hline " & $581-590$ & 8 \\
\hline " & $591-600$ & 8 \\
\hline 3 & $601-610$ & 98 \\
\hline “ & $\begin{array}{l}611-620 \\
621-630\end{array}$ & $\begin{array}{l}8 \\
9 \\
8\end{array}$ \\
\hline 4 & $631-640$ & 8 \\
\hline$u$ & $641-650$ & 8 \\
\hline & $651-660$ & 9.8 \\
\hline
\end{tabular}

\begin{tabular}{|c|c|c|c|c|}
\hline $\begin{array}{l}3 \\
43 \\
4.3 \\
3 \\
43\end{array}$ & $\begin{array}{llll}2 & 3.4 .5 & 6 \\
6 & & \\
6 & & \\
4 & 5 & 6 \\
7 & 6 & \end{array}$ & 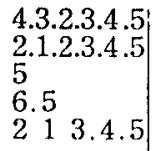 & $\begin{array}{lll}8 & 7 & \\
8 & 7 & \\
8 & 7 & \\
5 & 4 & 6.7 \\
8.7 & \end{array}$ & $\begin{array}{l}3.2 \\
3.2 \\
3.2 \\
3.2 \\
3.2\end{array}$ \\
\hline $\begin{array}{l}3 \\
4.3\end{array}$ & $\begin{array}{l}26 \\
76\end{array}$ & $\begin{array}{l}6.5 \\
6.5\end{array}$ & $\begin{array}{l}567 \\
7\end{array}$ & $\begin{array}{ll}3 & 2 \\
3 & 2\end{array}$ \\
\hline $\begin{array}{l}43 \\
43 \\
3 \\
43 \\
3 \\
3 \\
43 \\
4.4 \\
2.43 \\
3\end{array}$ & 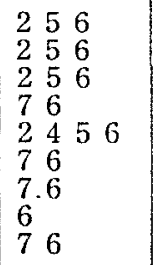 & 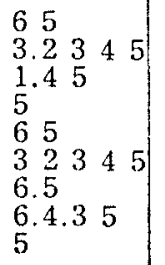 & $\begin{array}{ll}8 & 7 \\
8 & 7 \\
7 & \\
8 & 7 \\
8 & 7 \\
7 & \\
8 & 7 \\
7 & \\
7\end{array}$ & $\begin{array}{ll}1 & 3.2 \\
3 & 3.2 \\
2 & \\
3 & 2 \\
2 & \\
2 & \\
2 & \\
1 & 2 \\
3 & 2\end{array}$ \\
\hline 243 & 7.6 & 3.2 .65 & 87 & 2 \\
\hline $\begin{array}{l}43 \\
3\end{array}$ & $\begin{array}{l}23.456 \\
7.6\end{array}$ & $\begin{array}{l}65 \\
65\end{array}$ & $\begin{array}{ll}87 & \\
4 & 87\end{array}$ & $\begin{array}{ll}3 & 2 \\
1 & 3.2\end{array}$ \\
\hline $\begin{array}{ll}3 & \\
3 & \\
2.1 & 4.3\end{array}$ & $\begin{array}{ll}7 & 6 \\
7 & 6 \\
7.6\end{array}$ & $\begin{array}{llll}6.5 & & & \\
5 & & & \\
3 & 2.1 & 4 & 5\end{array}$ & $\begin{array}{llll}5 & 4 & 8 & 7 \\
8 & 7 & \\
8 & 7 & \end{array}$ & $\begin{array}{ll} & 3.2 \\
1 & 3.2 \\
3 & 2\end{array}$ \\
\hline $\begin{array}{l}4243 \\
3 \\
3 \\
43 \\
3 \\
3\end{array}$ & $\begin{array}{ll}7 & 6 \\
7 & 6 \\
4.56 & 6 \\
7 & 6 \\
7.6 \\
7.6\end{array}$ & $\begin{array}{lllll}6 & 5 & & & \\
5 & & & \\
6 & 4 & 3 & 6 & 5 \\
6 & 5 & & \\
4 & 5 & & \\
1 & 5 & & & \end{array}$ & $\begin{array}{l}87 \\
87 \\
87 \\
687 \\
87 \\
7\end{array}$ & $\begin{array}{l}2 \\
3.1 .2 \\
3.2 \\
3.2 .2 \\
3.2 \\
3.2\end{array}$ \\
\hline 2.43 & $\begin{cases}5 & 4.3 .2 \\
4 & 5 \\
6\end{cases}$ & 65 & 7 & 32 \\
\hline 3 & $\begin{array}{l}4 \\
5\end{array}$ & 24.5 & $\left\{\begin{array}{ll}8 & 6.5 .4 \\
7\end{array} 6.4\right.$ & $\begin{array}{lll}1 & 3 & 1.2\end{array}$ \\
\hline
\end{tabular}

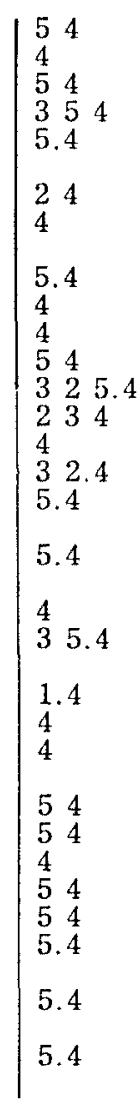

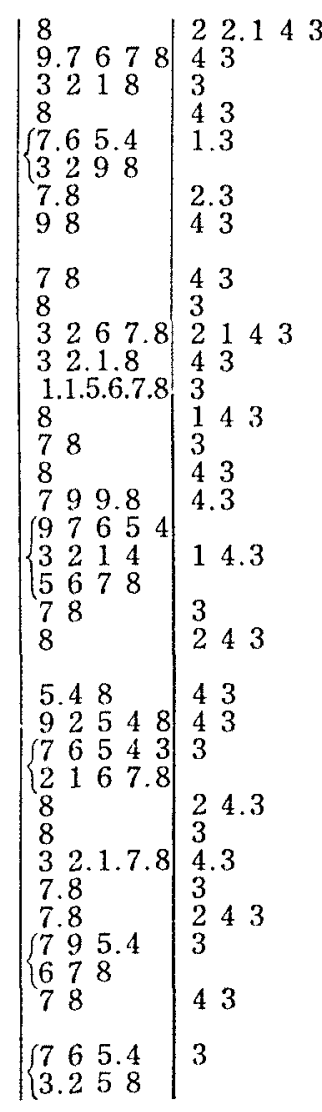

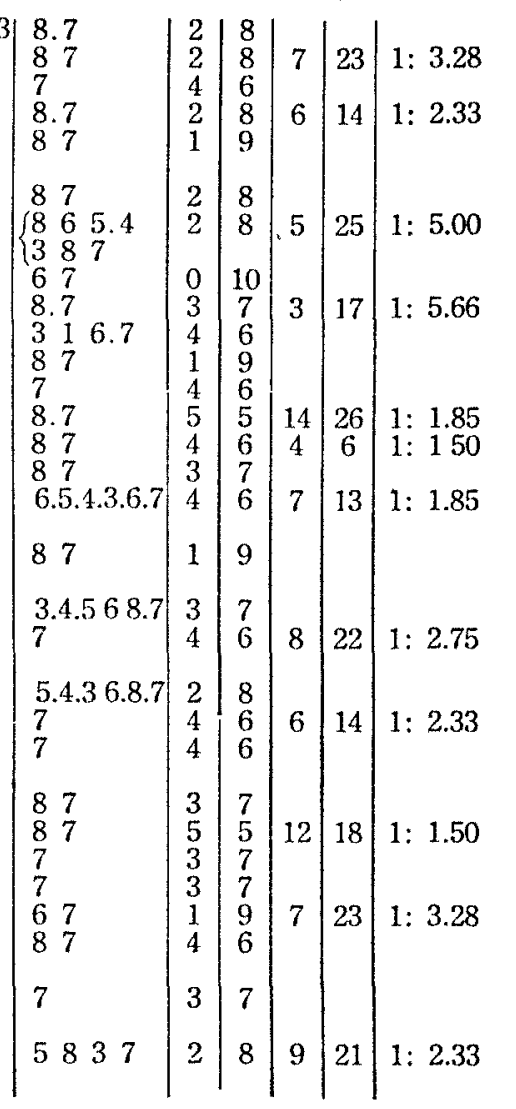


TABLE V-Continued

Results for Rat C, inbred Female, in Problem 2

\begin{tabular}{|c|c|c|c|c|c|c|c|c|c|c|c|c|c|c|c|c|}
\hline Date & $\begin{array}{c}\text { No. } \\
\text { of } \\
\text { trials }\end{array}$ & $\begin{array}{l}\text { S. } 1 \\
7.8 .9\end{array}$ & $\begin{array}{c}\text { S. } 2 \\
12.3 .4\end{array}$ & $\begin{array}{c}\text { S. } 3 \\
2.3 .4 .5 .6 .7\end{array}$ & $\begin{array}{c}\text { S. } 4 \\
1.2 .3 .4 .5 .6\end{array}$ & $\begin{array}{c}\text { S. } 5 \\
4.5 .6 .7 .8\end{array}$ & $\begin{array}{c}\text { S. } 6 \\
1.2 .3\end{array}$ & $\begin{array}{c}\text { S. } 7 \\
2.3 .4 .5\end{array}$ & $\begin{array}{c}\text { S. } 8 \\
1.2 .3 .4 .5 \\
67.8 .9\end{array}$ & $\begin{array}{c}\text { S. } 9 \\
1.2 .3 .4\end{array}$ & $\begin{array}{c}\text { S. } 10 \\
3.4 .5 .6 .7 .8\end{array}$ & $\mathrm{R}$ & W & $\mathrm{R}$ & $w$ & $\begin{array}{l}\text { Ratio } \\
\text { of } \\
\mathrm{R} \text { to } \mathrm{W}\end{array}$ \\
\hline $\begin{array}{c}\text { Sept. } \\
5 \\
" \\
" \\
7\end{array}$ & $\begin{array}{l}661-670 \\
671-680 \\
681-690 \\
691-700\end{array}$ & $\begin{array}{l}8 \\
78 \\
7.98 \\
8\end{array}$ & $\begin{array}{lll}4.3 & & \\
4 & 3 \\
4 & 2.1 & \\
3 & & \end{array}$ & $\begin{array}{rrrr}7 & 6 \\
7 & 6 & \\
7 & 6 & & \\
7 & 5 & 2 & 4\end{array}$ & $\begin{array}{llll}6 & 5 & \\
1 & 3 & 2 & 5 \\
6 & 5 & & \\
5 & & \end{array}$ & $\begin{array}{l}7 \\
7 \\
7 \\
47\end{array}$ & $\begin{array}{llll}3 & 2 & & \\
2 & & & \\
1 & 3 & 1 & 2 \\
3 & 2 & & \end{array}$ & 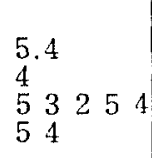 & $\begin{array}{lllll}8 & & & & \\
3 & 1 & 6 & 7 & 8 \\
8 & & & & \\
6 & 8 & & & \end{array}$ & $\begin{array}{ll}4 & 3 \\
4 & 3 \\
4 & 3 \\
4 & 3\end{array}$ & $\begin{array}{l}87 \\
7 \\
8.7 \\
84.37\end{array}$ & $\begin{array}{l}3 \\
4 \\
2 \\
3\end{array}$ & $\begin{array}{l}7 \\
6 \\
8 \\
7\end{array}$ & $\begin{array}{l}9 \\
3\end{array}$ & $\begin{array}{l}21 \\
7\end{array}$ & $\begin{array}{l}1: 2.33 \\
1: 2.33\end{array}$ \\
\hline $\begin{array}{l}8 \\
" \\
"\end{array}$ & $\begin{array}{l}701-710 \\
711-720 \\
721-730\end{array}$ & 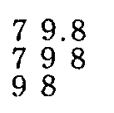 & $\begin{array}{l}3 \\
43 \\
3\end{array}$ & $\begin{array}{llll}7 & 2 & 4 & 6 \\
6 & & & \\
7 & 6\end{array}$ & $\begin{array}{llll}6 & 6 & 5 & \\
1 & 2 & 6 & 5 \\
6 & 5 & & \end{array}$ & $\begin{array}{l}87 \\
77 \\
87\end{array}$ & $\begin{array}{ll}3 & 2 \\
3 & 2 \\
3,2\end{array}$ & $\begin{array}{l}54 \\
44 \\
54\end{array}$ & $\begin{array}{llll}7 & 8 & & \\
8 & & & \\
7 & 6 & 5 & 4\end{array}$ & $\begin{array}{ll}3 & \\
4 & 3 \\
4 & 3\end{array}$ & $\begin{array}{ll}8 & 7 \\
8 & 7 \\
8 & 7\end{array}$ & $\begin{array}{l}2 \\
4 \\
1\end{array}$ & $\begin{array}{l}8 \\
6 \\
9\end{array}$ & & & \\
\hline “ & $\begin{array}{l}731-740 \\
741-750\end{array}$ & $\begin{array}{l}8 \\
8\end{array}$ & $\begin{array}{l}3 \\
243\end{array}$ & $\begin{array}{l}7.6 \\
7.6\end{array}$ & $\frac{4}{5} 3245$ & $\begin{array}{l}87 \\
57\end{array}$ & $\begin{array}{l}2 \\
2\end{array}$ & $\begin{array}{llll}3 & 2 & 5 & 4 \\
3 & 2 & 5 & 4\end{array}$ & $\left\{\begin{array}{lllll}7 & 8 & & \\
2 & 5 & 4 & 3 & 2\end{array}\right.$ & $\begin{array}{lll}2 & 1 & 4.3 \\
4 & 3\end{array}$ & $\begin{array}{l}87 \\
87\end{array}$ & $\begin{array}{l}3 \\
3\end{array}$ & $\begin{array}{l}7 \\
7\end{array}$ & 10 & 30 & $1: 3.00$ \\
\hline $\begin{array}{r}" 1 \\
10 \\
11\end{array}$ & $\begin{array}{l}751-760 \\
761-770 \\
771-780 \\
781-790 \\
791-800\end{array}$ & $\begin{array}{lll}8 & & \\
8 & & \\
9 & 7 & 8 \\
8 & 7 \\
8 & & \end{array}$ & 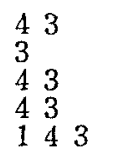 & 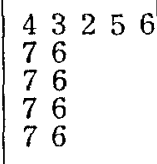 & $\begin{array}{ll}6 & 5 \\
6 & 5 \\
6 & 5 \\
6 & 5 \\
1.3 .6 & 2.6 .5 \\
\end{array}$ & $\begin{array}{lll}8 & 7 \\
7 & & \\
8 & 7 & \\
8 & 8 & 7 \\
8 & 7\end{array}$ & 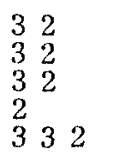 & $\begin{array}{ll}5 & 4 \\
5 & 2.4 \\
5 & 4 \\
5 & 4 \\
5 & 4\end{array}$ & $\begin{array}{l}9 \\
9 \\
8 \\
8 \\
8 \\
8 \\
8\end{array}$ & 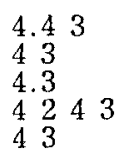 & $\begin{array}{l}7 \\
7 \\
87 \\
7 \\
87\end{array}$ & $\begin{array}{l}2 \\
5 \\
1 \\
3 \\
2\end{array}$ & $\begin{array}{l}8 \\
5 \\
9 \\
7 \\
8\end{array}$ & $\begin{array}{l}4 \\
2\end{array}$ & $\begin{array}{r}16 \\
8\end{array}$ & $\begin{array}{l}1: 200 \\
1: 4.00 \\
1: 4.00\end{array}$ \\
\hline
\end{tabular}


TABI.E. VI

RFsults FOR RAT B, OUTBREN FEMAIE, IN I'ROBLEM 2

\begin{tabular}{|c|c|c|c|c|c|c|c|c|c|c|c|c|c|c|c|c|}
\hline Date & $\begin{array}{l}\text { No. } \\
\text { of } \\
\text { trials }\end{array}$ & $\begin{array}{l}\text { S. } 1 \\
78.9\end{array}$ & $\begin{array}{c}S 2 \\
1.23 .1\end{array}$ & $\begin{array}{c}\text { S 3 } \\
3.4 .5 .6 .7\end{array}$ & $\begin{array}{c}\text { S. } 4 \\
12.3 .45 .6\end{array}$ & $\begin{array}{c}\text { S. } 5 \\
456.7 .8\end{array}$ & $\begin{array}{l}\text { S. } 6 \\
12.3\end{array}$ & $\begin{array}{c}\text { S. } 7 \\
23.4 .5\end{array}$ & $\begin{array}{c}S 8 \\
12.34 .5 \\
678.9\end{array}$ & $\begin{array}{c}\text { S. } 9 \\
1.2 .3 .4\end{array}$ & $\begin{array}{c}\$ 10 \\
3.4 .5 .6 .7 .8\end{array}$ & $\mathbf{R}$ & W & $\mathrm{R}$ & W & $\begin{array}{l}\text { Ratio } \\
\text { of } \\
R \text { to W }\end{array}$ \\
\hline June 30 & $1-6$ & 7778 & $\left\{\begin{array}{lllll}4 & 2 & 1 & 1 & 2 \\
1 & 2 & 2 & 2 & 3\end{array} \mid\right.$ & $\left|\begin{array}{lllll}2 & 2 & 2 & 2 & 2 \\
2 & 2 & 7 & *\end{array}\right|$ & & & 2 & & & & & 1 & 5 & 1 & 5 & $1: 5.00$ \\
\hline$\underset{1}{J u l y}$ & 7- 13 & 8 & 43 & $\left\{\begin{array}{llll}2 & 2 & 3 & 5 \\
3 & 6 & & \end{array}\right.$ & $\left\{\begin{array}{lllll}6 & 2 & 2 & 1 & 1 \\
1 & 4 & 1 & 3 & 5\end{array} \mid\right.$ & $\left\{\begin{array}{lllll}1 & 6 & 4 & 6 & 8 \\
4 & 1 & 8 & 6 & 7\end{array}\right.$ & 3112 & $\left\{\begin{array}{lllll}2 & 2 & 3 & 3 & 2 \\
3 & 2 & 3 & 3 & \\
2 & 2 & 5 & 4\end{array} \mid\right.$ & & & & 1 & 6 & 1 & 6 & $1: 600$ \\
\hline 2 & $14-23$ & 7798 & $\left\{\begin{array}{llll}4 & 2 & 1 & 4 \\
1 & 4 & 3\end{array}\right.$ & $\left\{\begin{array}{lllll}2 & 7 & 2 & 3 & 2 \\
7 & 2 & 3 & 7 \\
3 & 3 & 5 & 6\end{array}\right.$ & $\left\{\begin{array}{lllll}4 & 2 & 1 & 3 & 1 \\
2 & 3 & 1 & 6 & 1 \\
3 & 1 & 6 & 5 & \\
6 & 3 & 2 & 6 & 2\end{array} \mid\right.$ & $\left\{\begin{array}{llll}4 & 8 & 8 & 4 \\
4 & 6 & 8 & 7\end{array}\right.$ & $\begin{array}{lllll}3 & 1 & 1 & 3 & 2\end{array}$ & 324 & 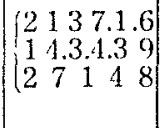 & $\begin{array}{lllll}1 & 1 & 1 & 1 & 3\end{array}$ & 8337 & 0 & 10 & 0 & 10 & 01000 \\
\hline 6 & $24-33$ & 78 & 3 & $\begin{array}{lllll}7 & 2 & 2 & 4 & 6\end{array}$ & $\mid \begin{array}{lllll}1 & 6 & 1 & 4 & 3 \\
1 & 2 & 6 & 1 & 4 \\
1 & 1 & 3 & 1 & 6\end{array}$ & $\left\{\begin{array}{llll}8 & 4 & 8 & 4 \\
4 & 4 & 7\end{array}\right.$ & 312 & 54 & 2578 & $\begin{array}{lllll}4 & 1 & 1 & 4 & 3\end{array}$ & $\begin{array}{lllll}5 & 4 & 3 & 6 & 7\end{array}$ & 1 & 9 & 1 & 9 & 19.00 \\
\hline 7 & $34-43$ & $\left\{\begin{array}{llll}7 & 7 & 79 \\
7 & 8 & \end{array}\right.$ & $\left\{\begin{array}{llll}4 & 2 & 1 & 4 \\
1 & 3 & & \end{array}\right.$ & $\left\{\begin{array}{lllll}2 & 4 & 2 & 4 & 7 \\
4 & 3 & 4 & 5 & 6\end{array}\right\}$ & $\left\{\begin{array}{lllll}1 & 2 & 4 & 1 \\
4 & 3 & 2 & 4 & 6 \\
1 & 3 & 1 & 5\end{array}\right.$ & 87 & $\left\{\begin{array}{llll}1 & 3 & 1 & 1 \\
1 & 2 & & \end{array}\right.$ & $\left\{\begin{array}{llll}2 & 2 & 3 & 5 \\
3 & 2 & 3 & 4\end{array}\right.$ & 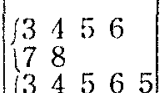 & 213 & $\left\{\begin{array}{llll}5 & 4 & 5 & 4 \\
5 & 6 & 7\end{array}\right.$ & 0 & 10 & 0 & 10 & $0 \cdot 10.00$ \\
\hline 8 & $44-53$ & $\left\{\begin{array}{llll}7 & 7 & 9 & 7 \\
9 & 7 & 8\end{array}\right.$ & 4123 & 776 & $\left\{\begin{array}{lllll}4 & 3 & 4 & 3 & 2 \\
1 & 3 & 4 & 5\end{array}\right.$ & 467 & 32 & 232334 & $\left|\begin{array}{lllll}3 & 4 & 0 & 0 & 0 \\
3 & 5 & 6 & 9 & 1 \\
3 & 9 & 9 & 1 & 8 \\
4 & 3 & 4 & 3 & 5 \\
4 & 3 & 2 & 3 & 4 \\
6 & 4 & 3 & 5 & 6\end{array}\right|$ & 43 & $\left\{\begin{array}{llll}3 & 5 & 3 & 4 \\
3 & 6 & 7\end{array}\right.$ & () & 10 & 0 & 10 & $0 \cdot 1000$ \\
\hline 9 & $54-63$ & 78 & $\left\{\begin{array}{llll}4 & 4 & 1 & 4 \\
2 & 1.3 & \end{array}\right.$ & 46 & 4345 & 467 & 2 & 54 & $\left|\begin{array}{lllll}4 & 5 & 6 & 3 & 9 \\
1 & 4 & 3 & 6 & 5 \\
4 & 1 & 3 & 7 & 1 \\
4 & 8 & & & \\
4 & 5 & 2 & 3.4 & 4\end{array}\right|$ & 13 & $\mid \begin{array}{lllll}3 & 3 & 6 & 8 & 6 \\
3 & 4 & 6 & 7\end{array}$ & 1 & 9 & 1 & 9 & $1 \cdot 9.00$ \\
\hline 10 & $61-73$ & $\left\{\begin{array}{llll}7 & 7 & 9 & 7 \\
7 & 8\end{array}\right.$ & 3 & $\left\{\begin{array}{llll}3 & 5 & 3 & 2 \\
5 & 4 & 5 & 6\end{array}\right.$ & 45 & 67 & 2 & $\left\{\begin{array}{llll}2 & 3 & 2 & 2 \\
3 & 4\end{array}\right.$ & $\left\{\begin{array}{lllll}2 & 3 & 4 & 5 & 2 \\
1 & 3 & 6 & 5 & 8\end{array} \mid\right.$ & 23 & 5347 & 2 & 8 & 2 & 8 & $1: 4.00$ \\
\hline 13 & $74-83$ & $\begin{array}{lllll}7 & 7 & 9 & 78\end{array}$ & 2423 & 2356 & $\left\{\begin{array}{llll}3 & 4 & 6 & 1 \\
2 & 1 & 6 & 5\end{array}\right.$ & 6447 & 2 & 31 & $\left|\begin{array}{llll}7 & 6 & 5 & 6 \\
7 & 8 & \end{array}\right|$ & 13 & $\mid \begin{array}{llll}5 & 3 & 4 & 5 \\
4 & 6 & 7 & \end{array}$ & $\begin{array}{l}1 \\
1\end{array}$ & $\stackrel{9}{9}$ & $\begin{array}{l}1 \\
1\end{array}$ & $\begin{array}{l}9 \\
9\end{array}$ & $\begin{array}{l}1: 9.00 \\
.19 .00\end{array}$ \\
\hline
\end{tabular}


TABLE VI-Continued

Results for Rat B, Outbred Female, Problem 2

\begin{tabular}{|c|c|c|c|c|c|c|c|c|c|c|c|c|c|c|c|c|}
\hline Date & $\begin{array}{l}\text { No. } \\
\text { of } \\
\text { trials }\end{array}$ & $\begin{array}{l}\text { S. } 1 \\
78.9\end{array}$ & $\begin{array}{c}\text { S. } 2 \\
1.2 .3 .4\end{array}$ & $\begin{array}{c}\text { S. } 3 \\
3.4 .5 .6 .7\end{array}$ & $\begin{array}{c}\text { S. } 4 \\
1.2 .3 .45 .6\end{array}$ & $\begin{array}{c}\text { S. } 5 \\
4.5 .67 .8\end{array}$ & $\begin{array}{l}\text { S. } 6 \\
1.2 .3\end{array}$ & $\begin{array}{c}\text { S. } 7 \\
23.4 .5\end{array}$ & $\begin{array}{c}\text { S. } 8 \\
1.2 .3 .4 .5 \\
6.7 .8 .9\end{array}$ & $\begin{array}{c}\text { S. } 9 \\
1.2 .3 .4\end{array}$ & $\begin{array}{c}\text { S. } 10 \\
3.4 .5 .6 .7 .8\end{array}$ & $R$ & $W$ & $\mathrm{R}$ & W & $\begin{array}{l}\text { Ratio } \\
\text { of } \\
R \text { to } W\end{array}$ \\
\hline July & $84-93$ & 78 & $\begin{array}{lllll}4 & 4 & 1 & 2 & 3\end{array}$ & 456 & 2345 & 7 & 3.1 .2 & 5.4 & $\left(\begin{array}{lllll}1 & 2 & 1 & 3 & 4\end{array} \mid\right.$ & 423 & 7 & 2 & 8 & & & \\
\hline$"$ & $94-103$ & 97.8 & 43 & $\left\{\begin{array}{lllll}3 & 2 & 3 & 4 & 5 \\
7 & 4 & 2 & 1 \\
2 & 4 & 5 & 6\end{array} \mid\right.$ & 3235 & 7 & 32 & 64 & 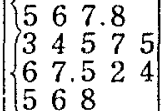 & 4423 & 3467 & 1 & 9 & 3 & 17 & $1: 5.66$ \\
\hline 15 & $\begin{array}{l}104-113 \\
114-123\end{array}$ & 7.7 .8 & 23 & 6 & $\begin{array}{l}3.45 \\
45\end{array}$ & $\begin{array}{l}4567 \\
567\end{array}$ & $\begin{array}{l}2 \\
3\end{array}$ & $\begin{array}{ll}5 & 4 \\
3 & 4\end{array}$ & $\left|\begin{array}{rrrrr}4 & 5 & 6 & 7 & 8 \\
7 & 5 & 6 & 7 & 8\end{array}\right|$ & $\begin{array}{llll}4 & 4 & 1 & 3\end{array}$ & 8.3 .4 .5 .6 .7 & $\begin{array}{l}2 \\
2\end{array}$ & 8 & 4 & $16>>$ & \\
\hline 16 & $\begin{array}{l}14-12 . \\
124-133\end{array}$ & 78 & $\begin{array}{ll}4 & 3 \\
2 & 3\end{array}$ & $\begin{array}{ll}450 \\
6\end{array}$ & $\begin{array}{ll}4 & 5 \\
6 & 65\end{array}$ & 567 & $\left\{\begin{array}{llll}3 & 3 & 2 & 3 \\
3 & 1 & 2\end{array}\right.$ & 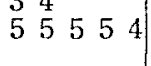 & $\begin{array}{llll}7 & 8 & 0\end{array}$ & 423 & $\left\{\begin{array}{lll}7 & 3 & 4.5 \\
6 & 7 & .5\end{array}\right)$ & $\begin{array}{l}2 \\
1\end{array}$ & $\begin{array}{l}8 \\
9\end{array}$ & 4 & 16 & $1: 4.00$ \\
\hline " & $134-143$ & 78 & $\left\{\begin{array}{llll}4 & 4 & 4 & 4 \\
4 & 4 & 3 & \end{array}\right.$ & 6 & 5 & 667 & $\left\{\begin{array}{lllll}3.3 .3 & 3.3 & 3 \\
3 & 3 & 3 & 3 & 2\end{array}\right.$ & $\left\{\begin{array}{llll}5 & 5 & 5 & 5 \\
5 & 2 & 3 & 4\end{array}\right.$ & 78 & $\left\{\begin{array}{llll}4 & 4 & 4 & 1 \\
2 & 3 & & \end{array}\right.$ & 7 & 3 & 7 & 4 & 16 & 1: 4.00 \\
\hline 17 & $144-153$ & 78 & 4423 & $\left\{\begin{array}{lllll}7 & 7 & 7 & 2 & 3 \\
2 & 3 & 4 & 5 & 6\end{array}\right.$ & 5 & $\begin{array}{llllll}8 & 8 & 5 & 6 & 7\end{array}$ & 3312 & $\begin{array}{lllll}5 & 3 & 2 & 3 & 3\end{array}$ & 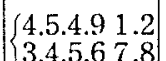 & $\begin{array}{rl}12 & 3 \\
4 & 3\end{array}$ & $\left\{\begin{array}{lll}8 & 8 & 8.5 \\
6 & 7 & \end{array}\right.$ & 1 & 9 & 1 & 9 & 1: 9.00 \\
\hline $\begin{array}{l}18 \\
19\end{array}$ & $\begin{array}{l}154-163 \\
164-173\end{array}$ & $\begin{array}{l}78 \\
78\end{array}$ & $\begin{array}{l}423 \\
3\end{array}$ & $\begin{array}{llll}6 & 3 & 4 & 5 \\
4 & 5 & 6\end{array}$ & $\begin{array}{llll}5 & & & \\
6 & 4 & 2 & 3\end{array}$ & $\begin{array}{rrrrr}5 & 4 & 5 & 6 & 7 \\
4 & 5 & 6 & 5\end{array}$ & $\begin{array}{llll}3 & 3 & 1 & 2 \\
3 & 3 & 1 & 2\end{array}$ & $\begin{array}{llll}5 & 5 & 2 & 3.4 \\
5 & 3 & 4\end{array}$ & $\left|\begin{array}{llll}3.4 .5 .0 & 6.8 \\
7 & 8 & & \\
5 & 6 & 4 & 5\end{array}\right|$ & $\begin{array}{lll}4 & 2 & 3 \\
4 & 2 & 3\end{array}$ & $\begin{array}{ll}8 & 7 \\
6 & 3.4 .5 .6 .7 \\
6 & 7\end{array}$ & $\begin{array}{l}2 \\
1\end{array}$ & $\begin{array}{l}8 \\
9\end{array}$ & 2 & 8 & $1: 4.00$ \\
\hline$a$ & $174-183$ & 8 & 443 & 723.45 .6 & $\begin{cases}4.5 \\
23 & 4.5\end{cases}$ & $\left\{\begin{array}{lll}6 & 7 & \\
5 & 6 & 7\end{array}\right.$ & 32 & 4 & $\left\{\begin{array}{lll}6 & 7 & 8 \\
7 & 8\end{array}\right.$ & 43 & - & 3 & 7 & 4 & 16 & 1: 4.00 \\
\hline 20 & $184-193$ & & 4423 & 56 & 45 & 57 & $\begin{array}{ll}3 & 2 \\
3 & 2\end{array}$ & $\begin{array}{l}4 \\
4\end{array}$ & $\mid \begin{array}{l}7 \\
78 \\
\end{array}$ & $\begin{array}{lllll}4 & 5 \\
4 & 4 & 1 & 2 & 3\end{array}$ & 7 & 3 & 7 & 4 & 10 & 1.4 .00 \\
\hline " & $194-203$ & 78 & 43 & 3456 & 45 & 67 & 32 & & $\begin{array}{lllll}4 & 5 & 6 & 7 & 8\end{array}$ & 43 & 567 & 1 & 9 & 4 & 16 & 1: 4.00 \\
\hline 21 & $204-213$ & 7.8 & 423 & & 45 & 567 & 32 & 34 & 78 & 43 & 56.7 & 1 & 9 & & & \\
\hline “ & $4-223$ & 78 & 43 & 456 & 4.3 .4 .6 .4 .5 & 867 & 2 & 234 & 5678 & 43 & & 2 & 8 & 3 & 17 & $1: 5.66$ \\
\hline 22 & $224-233$ & $78 \dagger$ & 43 & 456 & 5 & 67 & 32 & 4 & 458 & 3 & 567 & 3 & 7 & & & \\
\hline & $234-243$ & 78 & 43 & 456 & 5 & 5687 & 32 & 54 & $\left\{\begin{array}{llll}3 & 4 & 6 & 7 \\
1 & 3 & 6 & 8\end{array}\right.$ & 43 & 57 & 1 & 9 & 4 & 16 & $1: 4.00$ \\
\hline 23 & $244-253$ & 7978 & 443 & 4576 & 444435 & 457 & 32 & 24 & 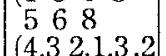 & 3 & 4.3 .4 .6 .5 .7 & 1 & 9 & & & \\
\hline " & $254-263$ & 978 & 23 & 46 & 45 & 44657 & 32 & 4 & 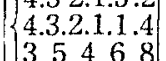 & 13 & 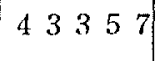 & 1 & 9 & 2 & 18 & 1: 9.00 \\
\hline 24 & $264-273$ & 8 & 3 & 576 & 465 & $\left\{\begin{array}{llll}4 & 6 & 5 & 4 \\
4 & 6 & 5 & 7\end{array}\right.$ & 32 & 4 & $\left\{\begin{array}{llll}5 & 4 & 3 & 2 \\
4 & 6 & 8\end{array} \mid\right.$ & 243 & 687 & 3 & 7 & 3 & 7 & 1: 2.33 \\
\hline
\end{tabular}




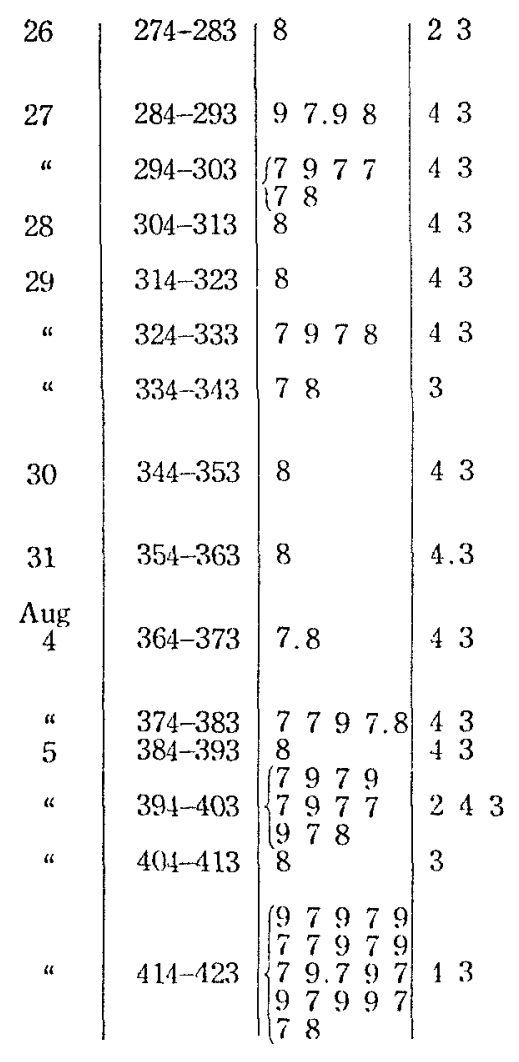

\begin{tabular}{|c|c|c|c|c|c|c|}
\hline 6 & 5 & 467 & 32 & 54 & $\left\{\begin{array}{llll}6 & 7 & 6 & 5 \\
0 & 1 & 8 & \end{array}\right.$ & 43 \\
\hline $\begin{array}{lllll}4 & 5 & 4 & 3 & 4 \\
3 & 2 & 4 & 3\end{array}$ & 65 & 47 & 32 & 54 & 468 & 43 \\
\hline 46 & 65 & 657 & $32^{\prime}$ & 54 & $\left\{\begin{array}{llll}4 & 3 & 2 & 1\end{array}\right.$ & 243 \\
\hline 6 & 5 & $\begin{array}{llll}4 & 4 & 6 & 8.7\end{array}$ & 32 & 54 & $\begin{array}{llll}5 & 4 & 3 & 4\end{array}$ & 43 \\
\hline 6 & 5 & $\begin{array}{llll}4 & 4 & 6 & 5.7\end{array}$ & 32 & 54 & 578 & 3 \\
\hline 6 & 5 & $\left\{\begin{array}{llll}5 & 4 & 6 & 5 \\
4 & 7\end{array}\right.$ & 32 & 4 & $\left\{\begin{array}{llll}9 & 4 & 3 & 2 \\
1 & 7 & 8\end{array}\right.$ & 43 \\
\hline 6 & 465 & 65437 & 32 & 24 & $\left\{\begin{array}{llll}5 & 4 & 3 & 6 \\
5 & 4 & 3 & 2\end{array}\right.$ & 3 \\
\hline$\left(\begin{array}{llll}5 & 4 & 3 & 2 \\
7 & 6 & & \end{array}\right.$ & 45 & $\left\{\begin{array}{llll}4 & 6 & 8 & 6 \\
8 & 7\end{array}\right.$ & 32 & 4 & $\left\{\begin{array}{llll}1 & 1 & 3 & 5 \\
7 & 6 & 8\end{array}\right.$ & 43 \\
\hline 551205 & 5 & $\left\{\begin{array}{llll}6 & 5 & 5 & 4 \\
6 & 5 & 4 & 7\end{array}\right.$ & 32 & 54 & 438 & 43 \\
\hline$\left|\begin{array}{lllll}5 & 4 & 3 & 2 & 5 \\
5 & 4 & 3 & 2 & 5 \\
5 & 4 & 3 & 2 . & 4 \\
3 & 5.4 & 4 & 6\end{array}\right|$ & 5 & 467 & 32 & 324 & $\left\{\begin{array}{lllll}4 & 3 & 2 & 4 \\
3 & 2 & 5 & 9 & 8\end{array} \mid\right.$ & 143 \\
\hline 6 & $\begin{array}{l}5 \\
3215\end{array}$ & $\begin{array}{l}47 \\
47\end{array}$ & $\begin{array}{ll}3 & 2 \\
3 & 2\end{array}$ & $\begin{array}{lllll}3 & 2 & 2 & 5 & 4 \\
4 & & & \end{array}$ & $\left|\begin{array}{lllll}5 & 9 & 1 & 6 & 8 \\
2 & 1 & 8 & & \end{array}\right|$ & $\frac{2}{3} 43$ \\
\hline 4.326 & 43265 & 47 & 32 & 324 & 8 & 43 \\
\hline$\left\{\begin{array}{lll}5 & 4 & 3.4 \\
3 & 6 & \end{array}\right.$ & 5 & 6547 & 32 & 3254 & $\left\{\begin{array}{lllll}4 & 3 & 2 & 7 & 4 \\
4 & 3 & 2 & 6 & 8\end{array} \mid\right.$ & 3 \\
\hline 5432.6 & 3265 & 5.187 & 32 & 3254 & $\left\{\begin{array}{lllll}3 & 2 & 1 & 6 & 5 \\
4 & 3 & 2 & 8\end{array} \mid\right.$ & 43 \\
\hline
\end{tabular}

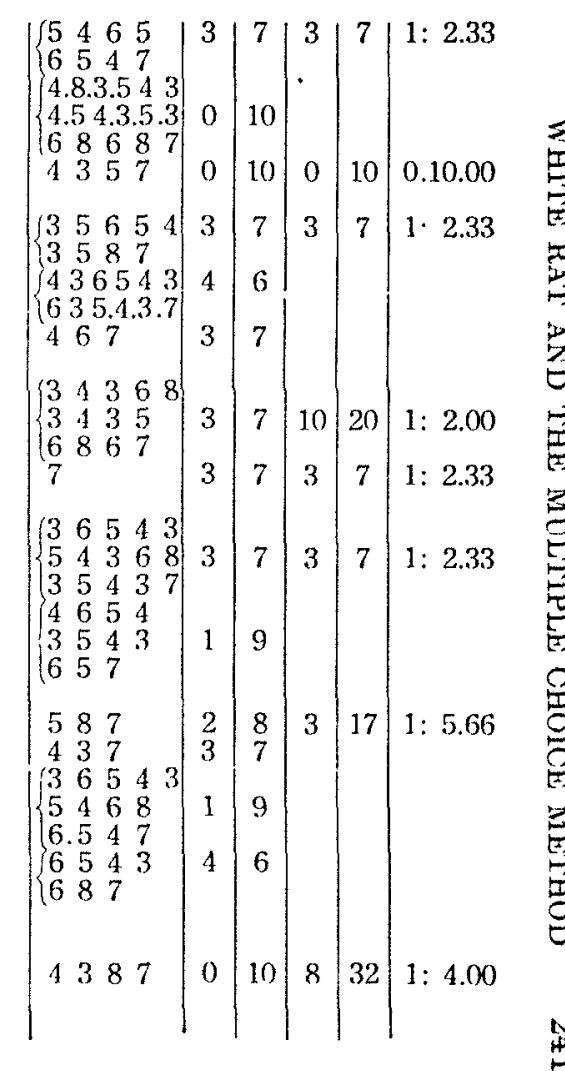


TABLE VI-Continued

Results for Rat B, Outbred Female, Problem 2

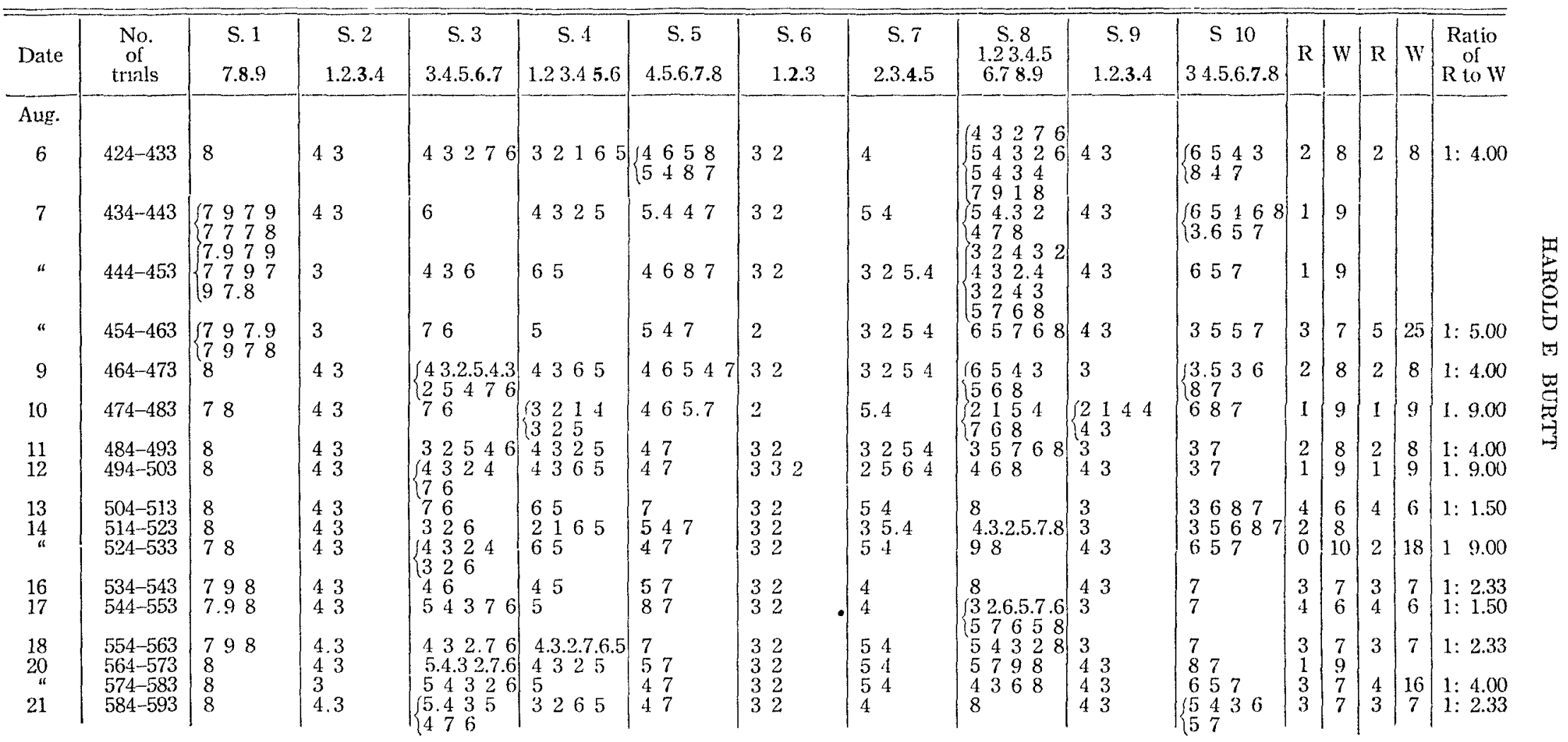




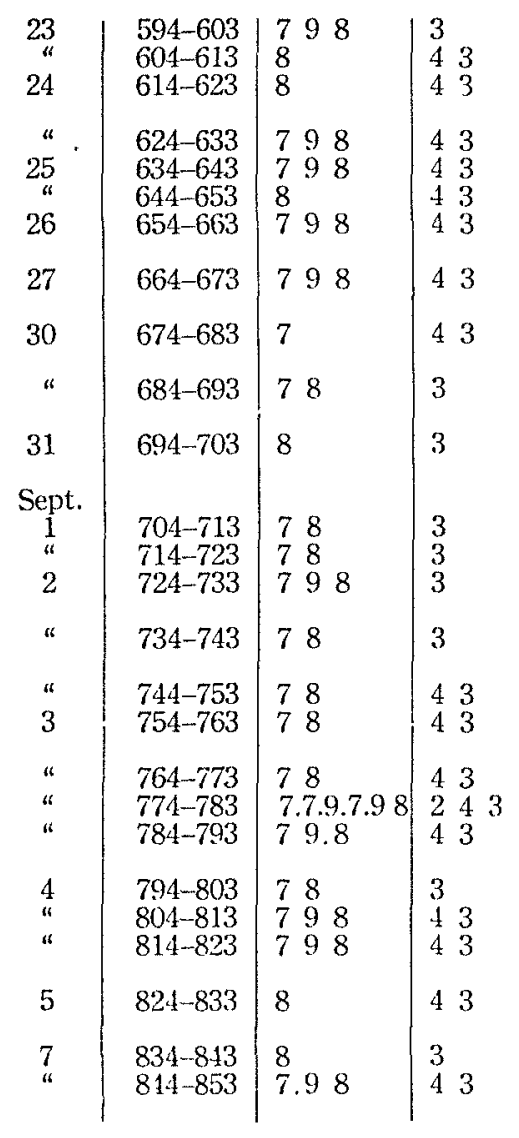

\begin{tabular}{|c|c|c|c|c|c|c|}
\hline $\begin{array}{lllll}3 & 2 & 5 & 7 & 6 \\
5 & 4 & 5 & 7 & 6 \\
4 & 3 & 2 & 5\end{array}$ & 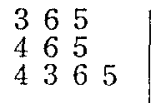 & $\begin{array}{llll}5 & 8 & 7 & \\
4 & 6 & 8 & 7 \\
6 & 8 & 9 & 7\end{array}$ & $\begin{array}{ll}1 & 3 \\
3 & 2 \\
3 & 2\end{array}$ & $\begin{array}{l}54 \\
44 \\
54\end{array}$ & $\begin{array}{lllll}7 & 9 & 7 & 6 & 8 \\
8 & & & \\
4 & 6 & 5 & 8\end{array}$ & \begin{tabular}{|ll}
4 & 3 \\
4 & 3 \\
4 & 3
\end{tabular} \\
\hline & 43265 & 587 & 32 & 4 & 7658 & 43 \\
\hline & & $\begin{array}{llll}4 & 7 & & \\
5 & 4 & 8 & 7\end{array}$ & $\begin{array}{ll}3 & 2 \\
3 & 2\end{array}$ & $\begin{array}{l}4 \\
54\end{array}$ & $\begin{array}{c}5.43229 .8 \\
7658\end{array}$ & $\begin{array}{ll}4 & 3 \\
4 & 3\end{array}$ \\
\hline 6 & $\left\{\begin{array}{llll}4 & 3 & 2 & 1 \\
6 & 5\end{array}\right.$ & 187 & 32 & 3254 & $\left\{\begin{array}{ccccc}4 & 3 & 2 & 7 & 6 \\
5 & 7 & 6 & 9 & 8\end{array}\right\}$ & 43 \\
\hline 436 & 43.265 & 547 & 32 & 3254 & 280 & 3 \\
\hline 3276 & 365 & 457 & 32 & 4 & $\begin{array}{lll}2 & 1 \\
0 & 0\end{array}$ & 43 \\
\hline 26 & 5 & $\left\{\begin{array}{llll}5 & 4 & 6 & 5 \\
4 & 8 & 7\end{array}\right.$ & 32 & 54 & 328 & 43 \\
\hline 76 & 6443655 & & 32 & 3254 & 15436 & 3 \\
\hline $\begin{array}{llll}5 & 4 & 3 & 5 \\
4 & 3 & 6\end{array}$ & 3265 & 47 & 32 & 254 & $\left\{\begin{array}{llll}3 & 6 & 5 & 7 \\
6 & 8 & 5 & \end{array}\right.$ & 43 \\
\hline $\begin{array}{l}54336 \\
2.6\end{array}$ & $\begin{array}{lll}2 & 5 & \\
3 & 6 & 5\end{array}$ & $\begin{array}{l}47 \\
4657 \\
465\end{array}$ & $\begin{array}{l}32 \\
2\end{array}$ & $\begin{array}{l}4 \\
3\end{array} 54$ & $8 \begin{array}{lll}8 & 5 & 4\end{array}$ & $\begin{array}{l}3 \\
3\end{array}$ \\
\hline 326 & 5 & 47 & 32 & 54 & 32.5435 & 43 \\
\hline $\begin{array}{l}576 \\
6\end{array}$ & $\begin{array}{llll}3 & 6 & 5 \\
4 & 3 & 6 & 5\end{array}$ & $\begin{array}{lllll}5 & 7 & & & \\
5 & 1 & 6 & 8 & 7\end{array}$ & $\begin{array}{l}2 \\
2\end{array}$ & $\begin{array}{l}54 \\
54\end{array}$ & $\begin{array}{lllll}7 & 8 & & & \\
9 & 1 & 5 & 7 & 8\end{array}$ & $\begin{array}{lll}1 & 4 & 3 \\
1 & 3\end{array}$ \\
\hline $\begin{array}{lllll}3 & 2 & 5 & 7 & 6 \\
6 & & & & \\
6 & & & & \end{array}$ & 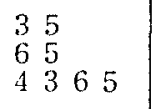 & $\begin{array}{llll}6 & 5 & 8 & 7 \\
4 & 4 & 7 \\
8 & 7 & \end{array}$ & $\begin{array}{ll}2 & \\
3 & 2 \\
3 & 2\end{array}$ & $\begin{array}{ll}5 & 4 \\
5 & 4 \\
5 & 4\end{array}$ & $\begin{array}{rr}8 & 1 \\
7 & 6 \\
4 & 3\end{array}$ & $\begin{array}{l}43 \\
143 \\
3\end{array}$ \\
\hline 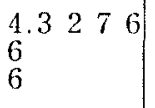 & $\begin{array}{lllll}3 & 2 & 6 & 5 \\
4 & 3 & 2 & 6 & 5 \\
4 & 3 & 2 & 6 & 5\end{array}$ & $\begin{array}{lll}6 & 5 & 7 \\
5 & 4 & 7 \\
4 & 7 & \end{array}$ & $\begin{array}{ll}3 & 2 \\
3 & 2 \\
3 & 2\end{array}$ & $\begin{array}{llll}3 & 2 & 5 & 4 \\
3 & 2 & 5 & 4 \\
3 & 2 & 5 & 4\end{array}$ & 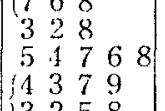 & $\begin{array}{ll}3 \\
4 & 3 \\
3\end{array}$ \\
\hline 4376 & 4365 & 57 & 32 & 54 & $\begin{array}{ll}8 & 1 \\
8 & 1\end{array}$ & 13 \\
\hline $\begin{array}{lllll}3 & 2 & 5 & 7 & 6 \\
6 & & & & \end{array}$ & $\begin{array}{lllll}1 & 3 & 2 & 6 & 5 \\
1 & 3 & 2 & 6 & 5\end{array} \mid$ & $\begin{array}{lll}5 & 4 & 7 \\
4 & 8 & 7\end{array}$ & $\begin{array}{ll}3 & 2 \\
3 & 2\end{array}$ & $\begin{array}{l}4 \\
4\end{array}$ & $\begin{array}{llll}6 & 1 & 5 & 4 \\
6 & 5 & 4 & 8\end{array}$ & $\begin{array}{ll}4 & 3 \\
4 & 3\end{array}$ \\
\hline
\end{tabular}

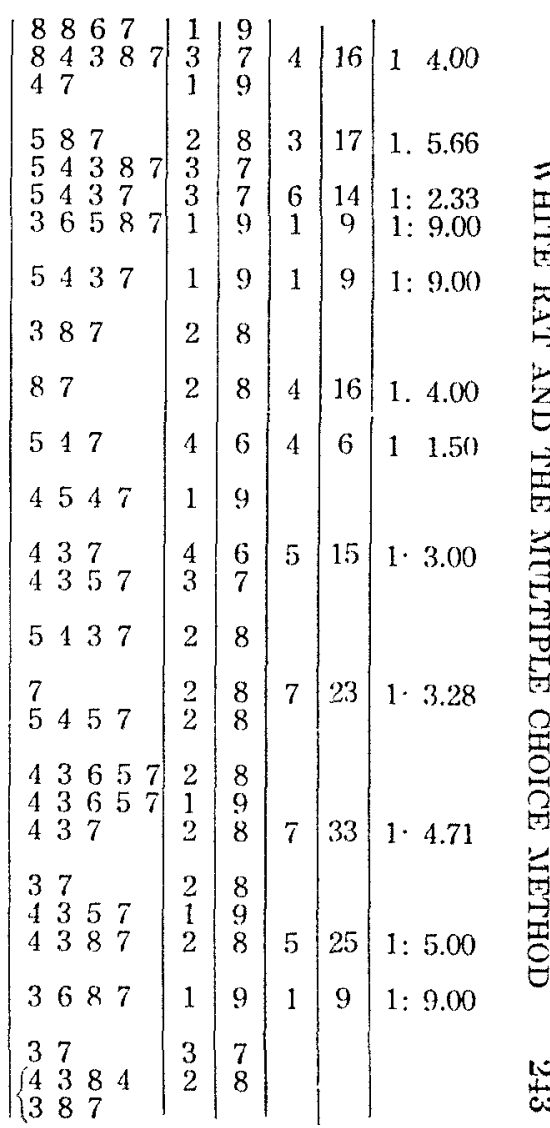


TABLE VI-Continued

\begin{tabular}{|c|c|c|c|c|c|c|c|c|c|c|c|c|c|c|c|c|}
\hline Date & $\begin{array}{l}\text { No. } \\
\text { of } \\
\text { trials }\end{array}$ & $\begin{array}{c}\text { S. } 1 \\
78.9\end{array}$ & $\begin{array}{c}\text { S. } 2 \\
12.3 .4\end{array}$ & $\begin{array}{c}\text { S. } 3 \\
34.56 .7 .\end{array}$ & $\begin{array}{c}\$ 4 \\
1.2 .3 .45 .6\end{array}$ & $\begin{array}{c}\text { S. } 5 \\
4.5 .67 .8\end{array}$ & $\begin{array}{l}\text { S. } 6 \\
1.2 .3\end{array}$ & $\begin{array}{c}\text { S. } 7 \\
2.3 .4 .5\end{array}$ & $\begin{array}{c}\text { S. } 8 \\
1.2345 \\
6.7 .8 .9\end{array}$ & $\begin{array}{c}\text { S. } 9 \\
123.4\end{array}$ & $\begin{array}{c}\text { S. } 10 \\
3.4 .5 .6 .7 .8\end{array}$ & $\mathbf{R}$ & $\mathrm{W}$ & $\mathrm{R}$ & W & $\begin{array}{l}\text { Ratio } \\
\text { of } \\
R \text { to } W\end{array}$ \\
\hline Sept & $854-863$ & 7.98 & 43 & 76 & 265 & 5487 & 32 & 3254 & $\left\{\begin{array}{llll}3 & 2 & 5 & 7 \\
6 & 5 & 7 & 8\end{array}\right.$ & 3 & 37 & 1 & 9 & 6 & 24 & 1: 4.00 \\
\hline 8 & $864-873$ & 7.9 .8 & 2143 & 436 & 3265 & 547 & 32 & 4 & $\left\{\begin{array}{llll}6 & 5 & 7 & 8 \\
4 & 3 & 2 & 7 \\
6 & 5 & 4 & 8\end{array}\right.$ & 143 & 87 & 1 & 9 & & & \\
\hline “ & $874-883$ & 798 & 3 & 3276 & 43265 & 5487 & 32 & 4 & $\left|\begin{array}{lllll}10 & 5 & 4 & 8 & \\
3 & 2 & 1 & 5 & 8\end{array}\right|$ & 143 & 37 & 2 & 8 & & & \\
\hline “ & $884-893$ & 798 & 3 & 6 & 65 & 547 & 32 & 53254 & $\left\{\begin{array}{llll}4 & 6 & 5 & 4 \\
3 & 6 & 7 & 8\end{array}\right\}$ & 243 & & 3 & 7 & 6 & 21 & $1: 4.00$ \\
\hline 9 & $894-903$ & 78 & 4 & 276 & 3265 & 587 & 32 & 4 & $\begin{array}{llll}1 & 0 & 1 & 8 \\
2 & 7 & 6 & 8\end{array}$ & 3 & 37 & 3 & 7 & 3 & 7 & 1: 2.33 \\
\hline
\end{tabular}

* Refused to work; laid down.

t Ten seconds punishment introduced 
a correct first choice in any trial is $1-5$ and the probable ratio or correct to incorrect first choices on a given day's trials, apart from experience, is 1 to 4 . With $\mathrm{C}$ this was exactly the ratio for the first day's trials. It was also the ratio for the last day's trials. $B$ started with a ratio of 1 to 5 which was reduced to 1 to 2.3 at the end. but there was no constancr in the results and the reactions were so manifestly random that further training seemed useless.

Although the rats failed to solve the problem, certain 1mportant reactive tendencies were noted. C hegan the problem 4 days after the check series on problem 1 The old halnt which was manifest at the outset disappeared gradually and was little in eridence after 100 trials. The tendency to enter the same (incorrect) door two or more times in succession also disappeared at about the same period in the training. Between trials 100 and 500 there was frequently a tendency to make several random choices and then work from right to left, entering consecutive doors successively. (cf, trials 133, 184, 338, 441 ). After this stage the tendency was not so manifest, for the first random choice was nearer the left. This latter fact also heightened the probability of a first correct random choice, which doubtless accounts for the higher scores in many of the later series. After trial 250 there began to appear a tendency to enter the left door and then the sccond from the lcft (cf. trials 251, 25t, 256, 257). This tendency was increasingly manifest throughout In trials 721-730 and 771-780, 8 out of 10 trials showed this type of reaction. The animal did show a definite tendency to turn to the left on leaving the entrance box, and often appeared to go directly to the door at the extreme left of those that were open. Whether it was merely this habit that was operative and the second choice naturally involved the neighboring door, or whether the rat formed the habit of going into the end compartment and then the second can not be definitely stated.

$B$ was set at problem 2 without prior training in problem 1 . Consequently there was no previous habit to break. B was likewise very active. At the outset the same door would be entered repeatedly (cf trial 3 ), but this was seldom the case after 150 trials. There soon appeared a tendency after an incorrect choice to enter alternate doors going rightward or to enter consecutive doors going leftward. Sometimes the one tendency was followed by the other. (cf. trials 167,268 ). After trial 270 there some- 
times appeared the opposite tendency, - to go leftward entering alternate or rightward entering consecutive ones. (cf. trial 271). These two tendencies to enter consecutive or alternate doors going in either direction were somewhat manifest throughout.

At trial 224 the period of punishment for B was increased to 10 seconds and soon after that she learned to stick her nose under the door (which was rased 3-16 inch from the floor to protect the tail), and wiggle out on her side before the 10 seconds were up. The direction in which she then turned appeared to depend much on her orientation as she came out of the compartment in this way. On the whole, B showed less systematic attack upon the problem than $\mathrm{C}$, choosing more palpably at random and getting fewer successes on the second choice.

\section{SUMMARY}

1. The first two standard multiple choice problems were presented to white rats These problems may be defined in terms of the constant relation of the correct mechanism to the varying group of mechanisms as: (1) the first at the right end of the series: (2) the second from the left end of the series.

2. One inbred and two outbred rats five months old solved the first problem in 200 trials or less. Another inbred individual two months younger required 350 trials. The indications are that visual tactual and kinaesthetic guidance sufficed for the formation of the habit.

The second problem proved insoluble for the two rats which attempted it. Both acquired a general tendency to turn toward the left, thus more frequently making a correct random choice, but the relation of secondness from the left was evidently beyond them

3. There were two noticeable reactive tendencies manifested by one rat. In problem 1 she would at times become oriented very suddenly, dash to the right end and then across to the correct door. In problem 2 she repeatedly entered the left door and then the second from the left. Although it is tempting to ascribe these tendencies to a higher level of behavior, it would seem better to explain the first in terms of kinaesthetic units and the second by the acquisition of the habit of choosing the left door,- the following choice most naturally involving the adjacent correct door. 